Research article

\title{
New approach for regulation of the internal recirculation flow rate by fuzzy logic in biological wastewater treatments
}

\author{
I. Santín ${ }^{\mathrm{a}, *}$, R. Vilanova ${ }^{\mathrm{a}}$, C. Pedret ${ }^{\mathrm{a}}$, M. Barbu ${ }^{\mathrm{a}, \mathrm{b}}$ \\ a Department of Telecommunications and Systems Engineering, School of Engineering, Universitat Autònoma de \\ Barcelona, 08193 Bellaterra, Barcelona, Spain \\ ${ }^{\mathrm{b}}$ Department of Automatic Control and Electrical Engineering, “Dunarea de Jos" University of Galati, 800008 Galati, Romania
}

\section{A R T I C L E I N F O}

\section{Article history:}

Received 7 August 2020

Received in revised form 17 March 2021

Accepted 17 March 2021

Available online 24 March 2021

\section{Keywords:}

Wastewater biological treatment Fuzzy control

Internal recirculation flow rate

Benchmark simulation model no 2

Control strategies

\begin{abstract}
A B S T R A C T
The internal recirculation plays an important role on the different biological processes of wastewater treatment plants because it has a great influence on the concentration of pollutants, especially nutrients. Usually, the internal recirculation flow rate is kept fixed or manipulated by control techniques to maintain a fixed nitrate set-point in the last anoxic tank. This work proposes a new control strategy to manipulate the internal recirculation flow rate by applying a fuzzy controller. The proposed controller takes into account the effects of the internal recirculation flow rate on the inlet of the biological treatment and on the denitrification and nitrification processes with the aim of reducing violations of legally established limits of nitrogen and ammonia and also reducing operational costs. The proposed fuzzy controller is tested by simulation with the internationally known benchmark simulation model no. 2. The objective is to apply the proposed fuzzy controller in any control strategy, only replacing the manipulation of the internal recirculation flow rate, to improve the plant operation.Therefore, it has been implemented in five operation strategies from the literature, replacing their original internal recirculation flow rate control, and simulation results are compared with those of the original strategies. Results show improvements with the application of the proposed fuzzy controller of between 2.25 and $57.94 \%$ in reduction of total nitrogen limit violations, between 55.22 and $79.69 \%$ in reduction of ammonia limit violations and between 0.84 and $38.06 \%$ in cost reduction of pumping energy.
\end{abstract}

(C) 2021 The Authors. Published by Elsevier Ltd on behalf of ISA. This is an open access article under the CC BY-NC-ND license (http://creativecommons.org/licenses/by-nc-nd/4.0/).

\section{Introduction}

Large amounts of freshwater are used daily around the world, becoming waste water. This waste water must be treated to avoid contamination of the receiving waters (rivers, lakes, etc.), which could affect aquatic life and consequently biodiversity. Due to this reason, wastewater treatment plants (WWTPs) are necessary to maintain the required levels of water quality.

Specifically, maximum concentration limits are established for discharges in the receiving environment of Total Suspended Solids (TSS), organic matter (Biological Oxygen Demand in 5 days $\left(B O D_{5}\right)$, and Chemical Oxygen Demand $(C O D)$, total nitrogen $\left(S_{\mathrm{Ntot}}\right)$, phosphorous and ammonium and ammonia nitrogen concentration $\left(S_{\mathrm{NH}}\right)$. Nitrogen and phosphorus are nutrients that can cause eutrophication in the receiving water, and consequently the death of aquatic beings. $S_{\mathrm{NH}}$, in addition to containing nitrogen, is toxic to aquatic life. Precisely, keeping $S_{\mathrm{Ntot}}$ and $S_{\mathrm{NH}}$

\footnotetext{
* Corresponding author.

E-mail addresses: Ignacio.Santin@uab.cat (I. Santín), Ramon.Vilanova@uab.cat (R. Vilanova), Carles.Pedret@uab.cat (C. Pedret), Marian.Barbu@ugal.ro (M. Barbu).
}

concentrations below the limits is usually one of the most difficult objectives to fulfill in WWTPs. In order to achieve all these targets, the application of operation strategies in WWTPs are very common.

Given the importance of keeping the pollutant concentration within the established limits, and achieving this with the lowest possible operational costs, several research works have been published in recent years focusing on the application of control strategies in WWTPs. In Katebi et al. [1], Santín et al. [2], i Arbós et al. [3] several control strategies applied in WWTPs are summarized.

Some works apply control strategies in the sludge treatment as in Barbu et al. [4] and Santín et al. [5], but most articles do it in the secondary treatment, which corresponds to biological treatment, whose operation is explained in Section 2. In the literature there are several works that aim to improve the control of the concentration of the dissolved oxygen $\left(S_{0}\right)$ in the aerobic reactors, by manipulating the oxygen transfer coefficient $\left(K_{\mathrm{L}} a\right)$, using different control techniques (Serralta et al. [6], Belchior et al. [7], Yang et al. [8], Harja et al. [9], Du et al. [10], Santín et al. [11]). The regulation of the $S_{0}$ set-point in the nitrification 


\section{List of abbreviations}

\begin{tabular}{|c|c|}
\hline $\mathrm{AE}$ & Aeration Energy (kWh/d) \\
\hline ASM1 & Activated Sludge Model no. 1 \\
\hline$B O D_{5}$ & 5-day Biological Oxygen Demand (mg/l) \\
\hline BSM1 & Benchmark Simulation Model no 1 \\
\hline BSM2 & Benchmark Simulation Model no2 \\
\hline$C O D$ & Chemical Oxygen Demand (mg/l) \\
\hline DCS & Default Control Strategy \\
\hline EC & External Carbon (kg/d) \\
\hline $\mathrm{HE}_{\text {net }}$ & Net Heating Energy $(\mathrm{kWh} / \mathrm{d})$ \\
\hline HRT & Hydraulic Retention Time (s) \\
\hline$K_{\mathrm{L}} a$ & Oxygen transfer coefficient $\left(\mathrm{d}^{-1}\right)$ \\
\hline$K_{\mathrm{L}} a_{\mathrm{i}}$ & $\begin{array}{l}\text { Oxygen transfer coefficient in tank } \mathrm{i} \\
\left(\mathrm{d}^{-1}\right)\end{array}$ \\
\hline ME & Mixing Energy $(\mathrm{kWh} / \mathrm{d})$ \\
\hline MET $_{\text {prod }}$ & $\begin{array}{l}\text { Methane production in the anaerobic } \\
\text { digester }(\mathrm{kg} / \mathrm{d})\end{array}$ \\
\hline MPC & Model Predictive Control \\
\hline $\mathrm{OCI}$ & Overall Cost Index \\
\hline$P E$ & Pumping Energy $(\mathrm{kWh} / \mathrm{d})$ \\
\hline PI & Proportional-Integral \\
\hline$Q$ & Flow rate $\left(\mathrm{m}^{3} / \mathrm{d}\right)$ \\
\hline$Q_{a}$ & Internal recycle flow rate $\left(\mathrm{m}^{3} / \mathrm{d}\right)$ \\
\hline$q_{\mathrm{EC}}$ & External carbon flow rate $\left(\mathrm{m}^{3} / \mathrm{d}\right)$ \\
\hline$q_{\mathrm{EC}, 1}$ & $\begin{array}{l}\text { External carbon flow rate in the first } \\
\text { tank }\left(\mathrm{m}^{3} / \mathrm{d}\right)\end{array}$ \\
\hline$Q_{\text {in }}$ & Influent flow rate $\left(\mathrm{m}^{3} / \mathrm{d}\right)$ \\
\hline$Q_{w}$ & Wastage flow rate $\left(\mathrm{m}^{3} / \mathrm{d}\right)$ \\
\hline$r_{\mathrm{S}_{\mathrm{NH}}}$ & $\begin{array}{l}\text { Conversion rate of ammonium and am- } \\
\text { monia nitrogen concentration in the } \\
\text { biological process }\end{array}$ \\
\hline$r_{\mathrm{S}_{\mathrm{NO}}}$ & $\begin{array}{l}\text { Conversion rate of nitrate concentration } \\
\text { in the biological process }\end{array}$ \\
\hline$S_{\text {Ntot }}$ & Total nitrogen concentration (mg/l) \\
\hline$S_{\text {Ntot,e }}$ & $\begin{array}{l}\text { Total nitrogen concentration in the } \\
\text { effluent }(\mathrm{mg} / \mathrm{l})\end{array}$ \\
\hline$S_{\mathrm{NH}}$ & $\begin{array}{l}\text { Ammonium and ammonia nitrogen } \\
\text { concentration }(\mathrm{mg} / \mathrm{l})\end{array}$ \\
\hline$S_{\mathrm{NH}, 0}$ & $\begin{array}{l}\text { Ammonium and ammonia nitrogen } \\
\text { concentration at the input of the first } \\
\text { reactor }(\mathrm{mg} / \mathrm{l})\end{array}$ \\
\hline$S_{\mathrm{NH}, 5}$ & $\begin{array}{l}\text { Ammonium and ammonia nitrogen } \\
\text { concentration at the output of the fifth } \\
\text { reactor }(\mathrm{mg} / \mathrm{l})\end{array}$ \\
\hline$S_{\mathrm{NH}, \text { in }}$ & $\begin{array}{l}\text { Ammonium and ammonia nitrogen } \\
\text { concentration in the influent }(\mathrm{mg} / \mathrm{l})\end{array}$ \\
\hline$S_{\mathrm{NH}, \mathrm{po}}$ & $\begin{array}{l}\text { Ammonium and ammonia nitrogen } \\
\text { concentration from the primary clarifier } \\
(\mathrm{mg} / \mathrm{l})\end{array}$ \\
\hline$S_{\mathrm{NH}, \mathrm{e}}$ & $\begin{array}{l}\text { Ammonium and ammonia nitrogen } \\
\text { concentration in the effluent }(\mathrm{mg} / \mathrm{l})\end{array}$ \\
\hline$S_{\mathrm{NO}}$ & Nitrate concentration $(\mathrm{mg} / \mathrm{l})$ \\
\hline$S_{\mathrm{NO}, 0}$ & $\begin{array}{l}\text { Nitrate concentration at the input of the } \\
\text { first reactor }(\mathrm{mg} / \mathrm{l})\end{array}$ \\
\hline$S_{\mathrm{NO}, 2}$ & $\begin{array}{l}\text { Nitrate concentration at the output of } \\
\text { the second reactor }(\mathrm{mg} / \mathrm{l})\end{array}$ \\
\hline$S_{\mathrm{NO}, 5}$ & $\begin{array}{l}\text { Nitrate concentration at the output of } \\
\text { the fifth reactor }(\mathrm{mg} / \mathrm{l})\end{array}$ \\
\hline$S_{0}$ & Dissolved oxygen concentration $(\mathrm{mg} / \mathrm{l})$ \\
\hline
\end{tabular}

$\begin{array}{ll}S_{\mathrm{O}, \mathrm{i}} & \begin{array}{l}\text { Dissolved oxygen concentration in tank } \\ \mathrm{i}(\mathrm{mg} / \mathrm{l})\end{array} \\ S P & \text { Sludge Production }(\mathrm{kg} / \mathrm{d}) \\ T_{\mathrm{as}} & \text { Temperature }\left({ }^{\circ} \mathrm{C}\right) \\ T S S & \text { Total Suspended Solids }(\mathrm{mg} / \mathrm{l}) \\ \text { WWTP } & \text { Wastewater Treatment Plants } \\ X_{\mathrm{B}, \mathrm{H}} & \text { The active heterotrophic biomass } \\ Z & \text { any concentration of the process } \\ Z_{\mathrm{i}} & \text { is } \mathrm{Z} \text { at the output of the reactor } \mathrm{i}\end{array}$

process has also been the goal of many articles, such as Serralta et al. [6], Vega et al. [12], Błaszkiewicz et al. [13], Santín et al. [14], Li et al. [15], Revollar et al. [16], Qiao et al. [17], Sadeghassadi et al. [18], Zhang et al. [19], Caraman et al. [20].

Another variable that can be manipulated in the biological treatment is the internal recirculation flow rate $\left(Q_{a}\right)$. However, research into a new control strategy to manipulate $Q_{a}$ is not common. The work Karches [21] shows the improvement obtained by adding an internal recirculation in completely stirred tank reactors. In some operation strategies of the articles cited above, $Q_{a}$ is kept fixed and in others $Q_{a}$ is manipulated to maintain the nitrate $\left(S_{\mathrm{NO}}\right)$ set-point in the last anoxic reactor. In Serralta et al. [6], Sadeghassadi et al. [18], Qiao et al. [17] and Caraman et al. [20], in addition to manipulating $S_{\mathrm{O}}$ set-point, the $S_{\mathrm{NO}}$ setpoint in the last anoxic reactor is regulated by manipulating $Q_{a}$ with different techniques. In Caraman et al. [20] by applying optimization techniques, in Sadeghassadi et al. [18] with neural network and MPC, in Qiao et al. [17] with adaptive fuzzy neural network and in Serralta et al. [6] by fuzzy controllers. In Serralta et al. [6] there are two internal recirculations in the biological treatment because anoxic and aerobic tanks are alternated. The works Revollar et al. [16] and Zhang et al. [19] apply optimization techniques to manipulate both $S_{\mathrm{O}}$ and $Q_{\mathrm{a}}$ directly, without $S_{\mathrm{NO}}$ control. In Santín et al. [22], the usual manipulation of $Q_{a}$ is modified to avoid $S_{\mathrm{NH}}$ violations, but only in the periods of time when a risk of $S_{\mathrm{NH}}$ violation is predicted.

Within the control techniques, it is common to find papers that apply fuzzy logic in WWTPs. Some of these articles have already been referred above, which regulate the $S_{\text {No }}$ set-point or $Q_{a}$ directly (Serralta et al. [6], Santín et al. [22], Qiao et al. [17], Caraman et al. [20]). In the case of Caraman et al. [20], the fuzzy controller is not applied to regulate $S_{\mathrm{O}}$ and $S_{\mathrm{NO}}$ set-points, but to differentiate the operating regime (dry, rain or storm). In addition to the articles mentioned, fuzzy controllers have also been applied in WWTPs for other purposes. For example, in Camilleri and Katebi [23] a fuzzy controller is applied for global control, taking into account not only WWTP but also the river and the sewer; and in Boiocchi et al. [24], the goal of the fuzzy controller is to reduce nitrous oxide emissions by manipulating the aeration of the aerobic reactor based on input and output values of $S_{\mathrm{NH}}$ and $S_{\text {NO. }}$

The novelty proposed in this paper is a new control strategy to manipulate $Q_{a}$ by a fuzzy controller. The variation of $Q_{a}$ has several effects on the nitrification and denitrification processes of the biological treatment. The analysis of these effects explains why the proposed control strategy has better results than those normally used. For the design of the Proposed Fuzzy Controller for $Q_{a}$ manipulation (PFC_ $Q_{a}$ ), the dilution or the increase of concentration of different compounds at the beginning of the biological treatment is taken into account, as well as the variation of the Hydraulic Retention Time (HRT) (a more exhaustive explanation is found in Section 3). This paper uses operation strategies from the literature, replacing only the manipulation of $Q_{a}$ by PFC_ $Q_{a}$, 


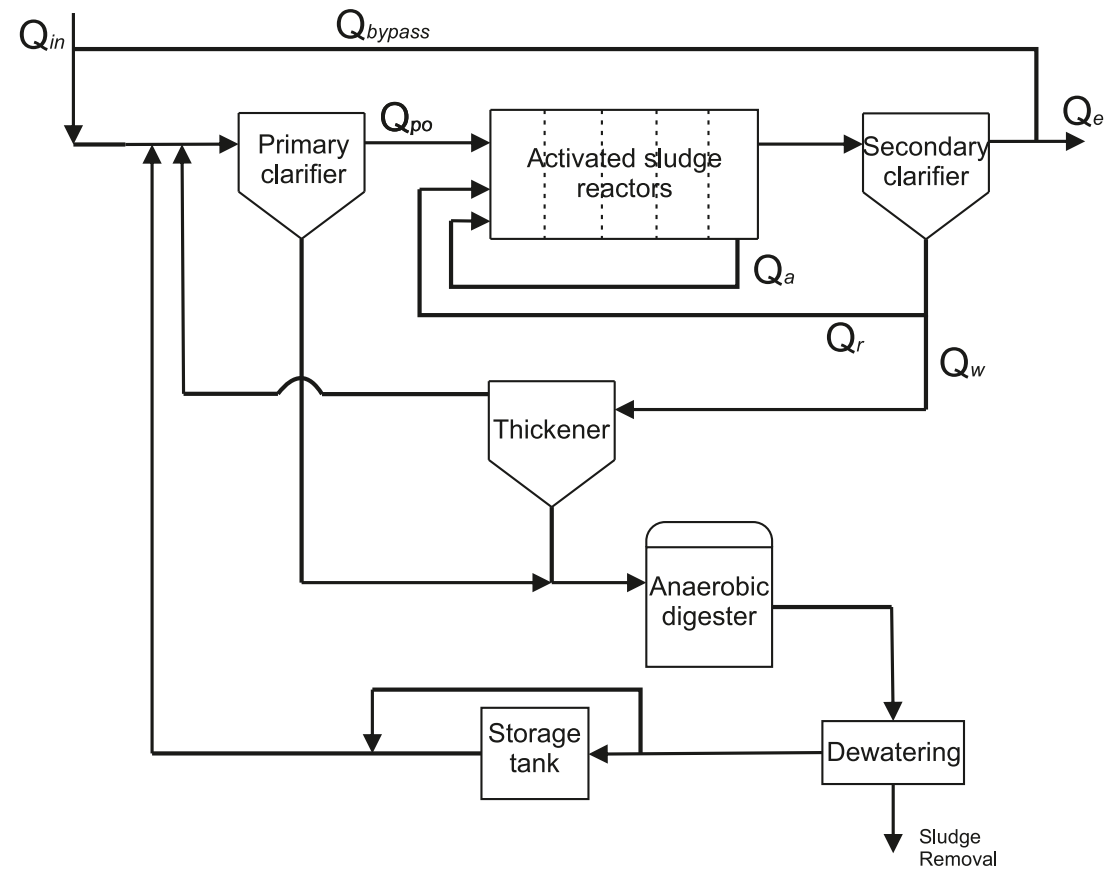

Fig. 1. BSM2 plant with notation used for flow rates.

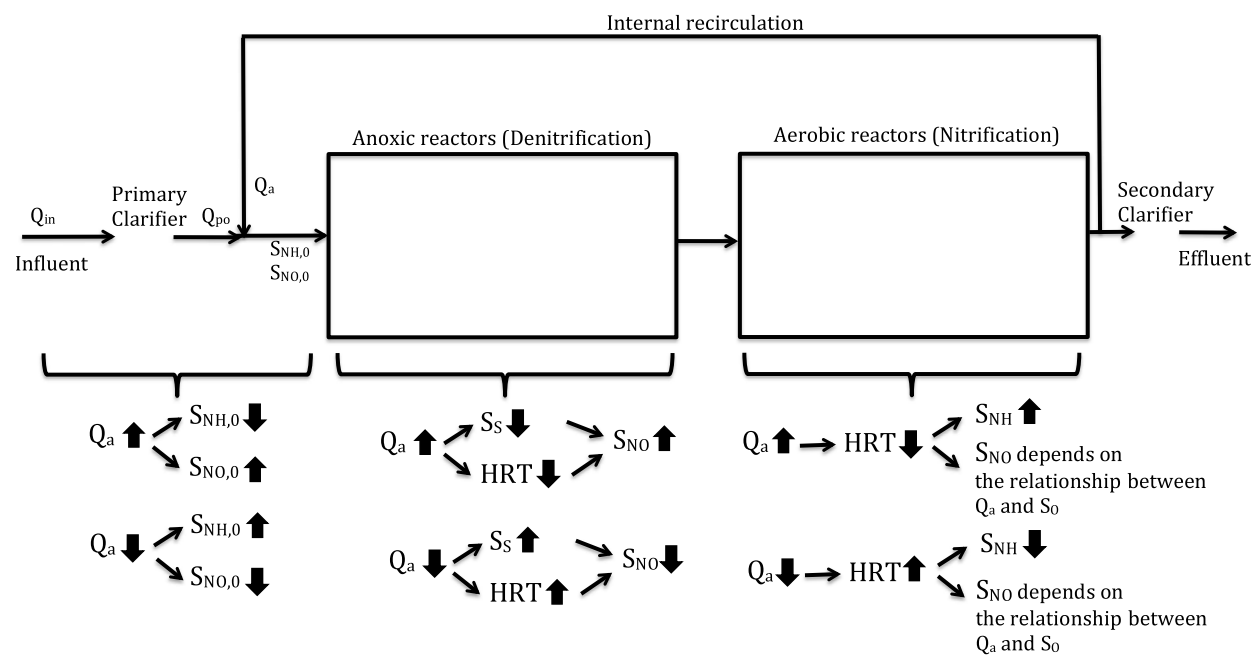

Fig. 2. $Q_{a}$ effects on the biological treatment.

and shows the improvements it provides in terms of reducing violations of $S_{\mathrm{Ntot}}$ and $S_{\mathrm{NH}}$, and operational costs.

The use of simulation models to test control strategies in WWTPs is a common practice in research. So much so that most of the previously referenced papers use the internationally known and accepted standard Benchmark Simulation Model no. 2 (BSM2) $^{1}$ (Gernaey et al. [25]), which is an extension of the Benchmark Simulation Model no. 1 (BSM1) developed by the International Association on Water Pollution Research and Control (Alex et al. [26], Copp [27]). BSM2 differs from BSM1 by including the entire cycle of a WWTP, adding the sludge treatment, and in that the simulation period is extended to oneyear assessment. In this work, the simulations and evaluations of the control strategies have been carried out with BSM2.

The paper is organized as follows. First, BSM2 is presented. Next, PFC_ $Q_{a}$ is explained. Afterward, the simulation results are

\footnotetext{
1 http://iwa-mia.org/benchmarking
}

shown, as well as the discussion about them. Finally, the most important conclusions are drawn.

\section{Materials and methods}

The evaluation of the PFC_ $Q_{a}$ for wastewater treatment plants has been carried out using the internationally known BSM2 (Jeppsson et al. [28]), which was updated by Nopens et al. [29]. The BSM2 layout (Fig. 1) includes the biological treatment (secondary treatment) of BSM1 and, in addition, a primary settler, a sludge treatment by a thickener, a digester and a dewatering. The liquid extracted in the dewatering is stored in a regulator tank and later recirculated to the primary settler.

BSM2 includes dynamics of the different influent concentrations for a period of 609 days, and the data from day 245 to 609 (one year) are evaluated. These dynamics include rain events and temperature $\left(T_{\mathrm{as}}\right)$ variations.

The biological treatment consists of five activated sludge reactors, followed by a secondary settler. The first two reactors 


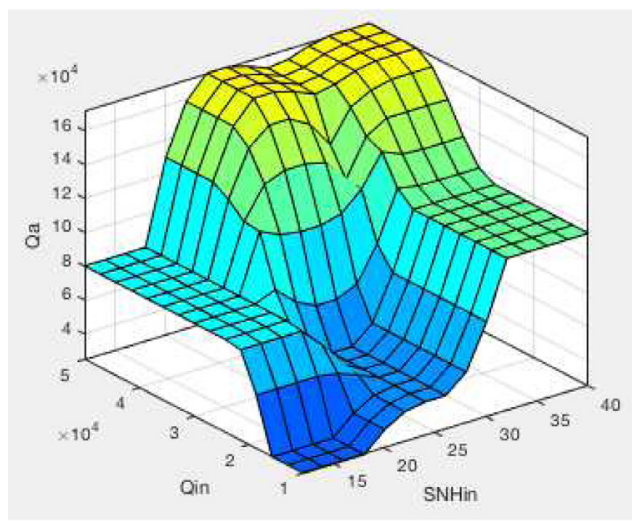

(a) X input: $Q_{\text {in }}$; Y input: $S_{\mathrm{NH}, \mathrm{in}}$; Z Output: $Q_{\mathrm{a}}$ with $S_{\mathrm{NH}, 0}$ low, $S_{\mathrm{NH}, 5}$ not high, $S_{\mathrm{NO}, 5}$ medium and $T_{\mathrm{as}}$ low and high $\left(15^{\circ} \mathrm{C}\right)$

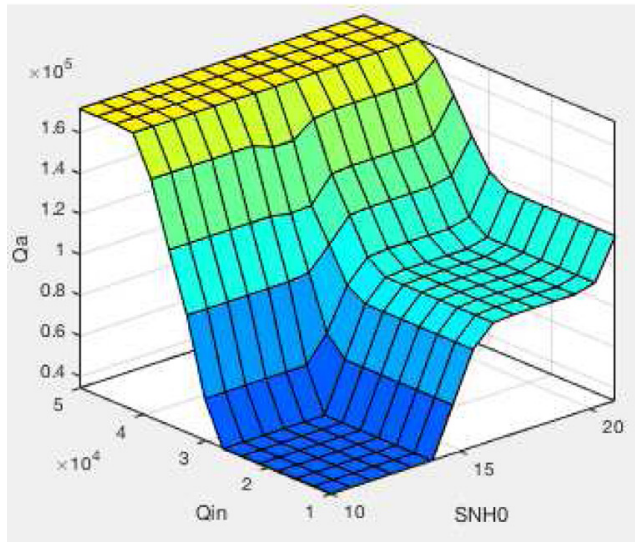

(c) X input: $Q_{\text {in }} ; \mathrm{Y}$ input: $S_{\mathrm{NH}, 0} ; \mathrm{Z}$ Output: $Q_{\mathrm{a}}$ with $S_{\mathrm{NH}, \text { in }}$ medium, $S_{\mathrm{NH}, 5}$ not high, $S_{\mathrm{NO}, 5}$ medium and $T_{\mathrm{as}}$ low and high $\left(15^{\circ} \mathrm{C}\right)$

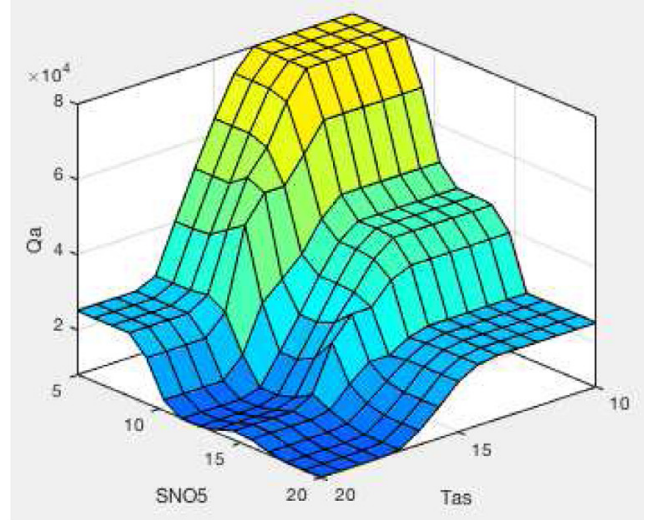

(b) X input: $S_{\mathrm{NO}, 5} ; \mathrm{Y}$ input: $T_{\mathrm{as}} ; \mathrm{Z}$ Output: $Q_{\mathrm{a}}$ with $S_{\mathrm{NH}, \text { in }}$ medium, $S_{\mathrm{NH}, 0}$ low, $S_{\mathrm{NH}, 5}$ not high and $Q_{\text {in }}$ low

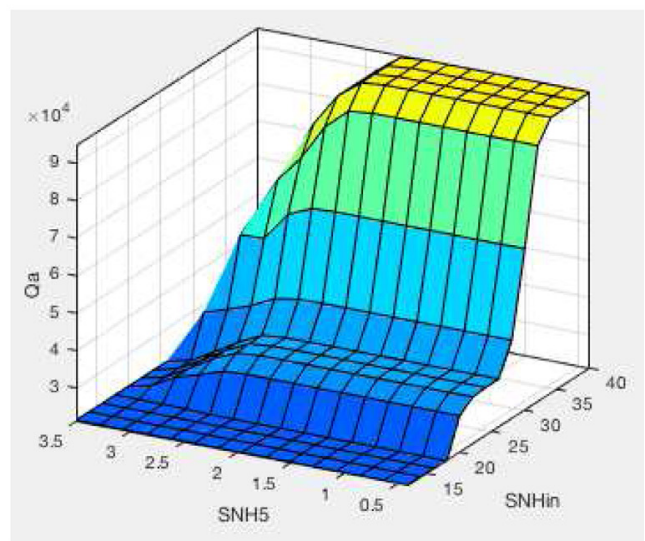

(d) X input: $S_{\mathrm{NH}, 5} ; \mathrm{Y}$ input: $S_{\mathrm{NH}, \text { in }}$; Z Output: $Q_{\mathrm{a}}$ with $S_{\mathrm{NH}, 0}$ low, $S_{\mathrm{NO}, 5}$ medium, $Q_{\text {in }}$ low and $T_{\text {as }}$ low and high $\left(15^{\circ} \mathrm{C}\right)$

Fig. 3. Graphic surfaces of the fuzzy control output related to the inputs.

are anoxic, where the denitrification process is carried out, and the following three tanks are aerobics, where the nitrification process takes place. The influent has an average dry weather flow rate of $20,648.36 \mathrm{~m}^{3} / \mathrm{d}$ and an average COD of $592.53 \mathrm{mg} / \mathrm{l}$. The volume of each anoxic tank is $1500 \mathrm{~m}^{3}$ and that of each aerobic tank is $3000 \mathrm{~m}^{3}$. The HRT of the biological treatment is $14 \mathrm{~h}$. There is a recirculation from the last tank to the first one (internal recirculation) and another from the underflow of the settler (external recirculation) in order to recirculate sludge.

The Activated Sludge Model No. 1 (ASM1) (Henze et al. [30]) describes the processes of the biological reactors. They define the conversion rates and the mass balance of the different variables of the biological treatment. The design of the PFC_ $Q_{a}$ is based on the $Q_{a}$ effects on the biological treatment taking into account the conversion rates of $S_{\mathrm{NH}}\left(r_{\mathrm{S}_{\mathrm{NH}}}\right)$ and $S_{\mathrm{NO}}\left(r_{\mathrm{S}_{\mathrm{NO}}}\right)$ and the reactors mass balance.

The equations of conversion rates and mass balance are shown below:

$r_{N H}=-0.08 \rho_{1}-0.08 \rho_{2}-\left(0.08+\frac{1}{0.24}\right) \rho_{3}+\rho_{6}$

$r_{N O}=-0.1722 \rho_{2}+4.1667 \rho_{3}$

where $\rho_{1}, \rho_{2}, \rho_{3}, \rho_{6}$ are four of the eight biological processes defined in ASM1. Specifically, $\rho_{1}$ is the aerobic growth of heterotrophs, $\rho_{2}$ is the anoxic growth of heterotrophs, $\rho_{3}$ is the aerobic growth of autotrophs and $\rho_{6}$ is the ammonification of soluble organic nitrogen. They are defined below:

$\rho_{1}=\mu_{H T}\left(\frac{S_{S}}{10+S_{S}}\right)\left(\frac{S_{O}}{0.2+S_{0}}\right) X_{B, H}$

where $S_{\mathrm{S}}$ is the readily biodegradable substrate and $\mu_{\mathrm{HT}}$ is:

$\mu_{H T}=4 \cdot \exp \left(\left(\frac{\operatorname{Ln}\left(\frac{4}{3}\right)}{5}\right) \cdot\left(T_{a s}-15\right)\right)$

$\rho_{2}=\mu_{H T}\left(\frac{S_{S}}{10+S_{S}}\right)\left(\frac{0.2}{0.2+S_{O}}\right)\left(\frac{S_{N O}}{0.5+S_{N O}}\right) 0.8 \cdot X_{B, H}$

$\rho_{3}=\mu_{A T}\left(\frac{S_{N H}}{1+S_{N H}}\right)\left(\frac{S_{O}}{0.4+S_{O}}\right) X_{B, A}$

where $X_{\mathrm{B}, \mathrm{A}}$ is the active autotrophic biomass and $\mu_{\mathrm{AT}}$ is:

$\mu_{A T}=0.5 \cdot \exp \left(\left(\frac{\operatorname{Ln}\left(\frac{0.5}{0.3}\right)}{5}\right) \cdot\left(T_{a s}-15\right)\right)$

$\rho_{6}=k_{a T} \cdot S_{N D} \cdot X_{B, H}$

where $S_{\mathrm{ND}}$ is the soluble biodegradable organic nitrogen and $k_{\mathrm{aT}}$ is:

$k_{a T}=0.05 \cdot \exp \left(\left(\frac{\operatorname{Ln}\left(\frac{0.05}{0.04}\right)}{5}\right) \cdot\left(T_{a s}-15\right)\right)$

The general equations for mass balancing are: 


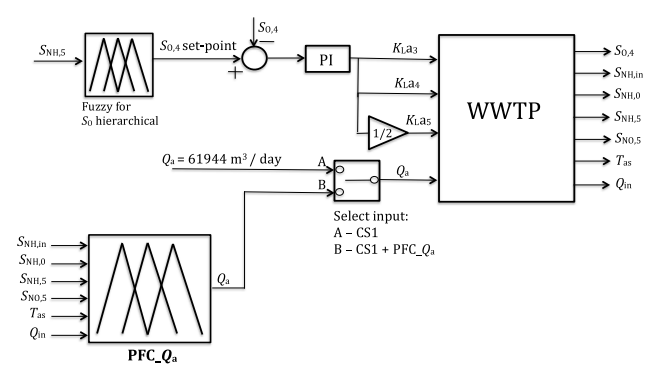

(a) CS1 (selection $\mathrm{A})$ and $\mathrm{CS} 1+\mathrm{PFC}-Q_{\mathrm{a}}($ selection $\mathrm{B})$

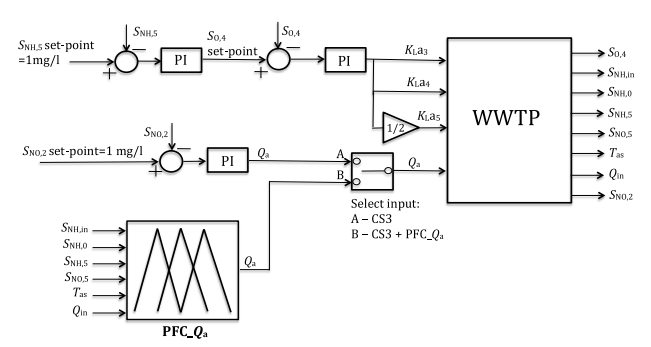

(c) $\mathrm{CS} 3$ (selection $\mathrm{A}$ ) and $\mathrm{CS} 3+\mathrm{PFC} Q_{\mathrm{a}}$ (selection $\left.\mathrm{B}\right)$

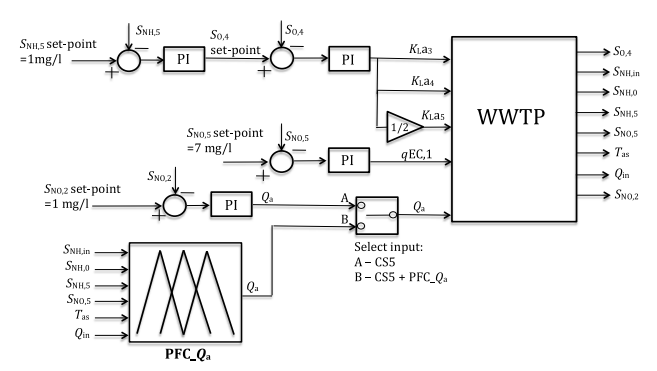

(e) CS5 (selection A) and CS5+PFC_ $Q_{\mathrm{a}}$ (selection B)

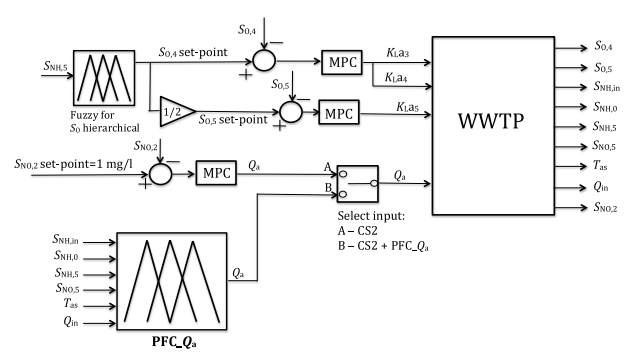

(b) $\mathrm{CS} 2$ (selection $\mathrm{A}$ ) and $\mathrm{CS} 2+\mathrm{PFC}_{-} Q_{\mathrm{a}}$ (selection $\left.\mathrm{B}\right)$

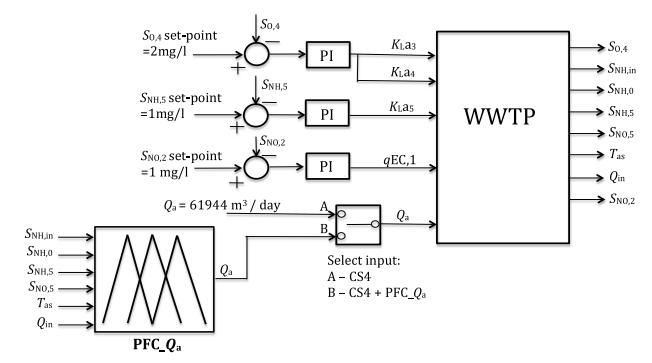

(d) CS4 (selection A) and CS4+PFC_ $Q_{\mathrm{a}}$ (selection B)

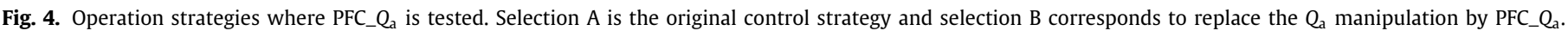

- For reactor 1:

$\frac{d Z_{1}}{d t}=\frac{1}{V_{1}}\left(Q_{a} \cdot Z_{a}+Q_{r} \cdot Z_{r}+Q_{p o} \cdot Z_{p o}+r_{z, 1} \cdot V_{1}-Q_{1} \cdot Z_{1}\right)$

where $Z$ is any concentration of the process, $Z_{1}$ is $Z$ in the first reactor, $Z_{\mathrm{a}}$ is $Z$ in the internal recirculation, $Z_{\mathrm{r}}$ is $Z$ in the external recirculation, $Z_{\mathrm{po}}$ is $Z$ from the primary clarifier, $\mathrm{V}$ is the volume, $V_{1}$ is $\mathrm{V}$ in the first reactor, $Q_{\mathrm{po}}$ is the overflow from the primary clarifier and $Q_{1}$ is the flow rate in the first tank and it is equal to the sum of $Q_{a}, Q_{r}$ and $Q_{p o}$.

- For reactor 2 to 5 :

$$
\frac{d Z_{k}}{d t}=\frac{1}{V_{k}}\left(Q_{k-1} \cdot Z_{k-1}+r_{z, k} \cdot V_{k}-Q_{k} \cdot Z_{k}\right)
$$

where $k$ is the number of reactor and $Q_{k}$ is equal to $Q_{k-1}$

Result evaluation is carried out by the effluent quality and the operational costs. Effluent quality is evaluated by the percentage of time that the effluent concentrations of $S_{\mathrm{Ntot}}, C O D, S_{\mathrm{NH}}$, TSS and $B O D_{5}$ are above the established limits, shown in Table A.6. Costs are evaluated by the Overall Cost Index (OCI) and each of its components.

OCI is defined to evaluate the operational cost as:

$O C I=A E+P E+3 \cdot S P+3 \cdot E C+M E-6 \cdot M E T_{\text {prod }}+H E_{\text {net }}$

where $A E$ is the aeration energy, $P E$ is the pumping energy, $S P$ is the sludge production to be disposed, $E C$ is the consumption of external carbon source, $M E$ is the mixing energy, $M E T_{\text {prod }}$ is the methane production in the anaerobic digester and $H E_{\text {net }}$ is the net heating energy.

$A E$ is calculated according to the following relation:

$A E=\frac{8}{T \cdot 1.8 \cdot 1000} \int_{t=245 \text { days }}^{t=609 \text { days }} \sum_{i=1}^{5} V_{i} \cdot K_{L} a_{i}(t) \cdot d t$

where $K_{L} a_{i}$ is $K_{L}$ a in tank $i$.

$P E$ is calculated as:

$$
\begin{aligned}
P E= & \frac{1}{T} \int_{245 \text { days }}^{609 \text { days }}\left(0.004 \cdot Q_{0}(t)+0.008 \cdot Q_{a}(t)\right. \\
& \left.+0.06 \cdot Q_{w}(t)+0.06 \cdot Q_{t o}(t)+0.004 \cdot Q_{d u}(t)\right) \cdot d t
\end{aligned}
$$

where $Q_{w}$ is the wastage flow rate, $Q_{\text {to }}$ is the overflow rate from the thickener and $Q_{d u}$ is the underflow rate.

$S P$ is calculated from the total solid flow from wastage and the solids accumulated in the system over the period of time considered:

$$
\begin{aligned}
S P= & \frac{1}{T} \cdot\left(\text { TSS }_{a}(609 \text { days })-\text { TSS }_{a}(245 \text { days })\right. \\
& + \text { TSS }_{s}(609 \text { days })-\text { TSS }_{S}(245 \text { days })+ \\
& \left.+0.75 \cdot \int_{t=245 \text { days }}^{t=609 \text { days }} \operatorname{TSS}_{w} \cdot Q_{w} \cdot d t\right)
\end{aligned}
$$

where $T S S_{a}$ is the amount of solids in the reactors, $T S S_{\mathrm{s}}$ is the amount of solids in the settler and $T S S_{\mathrm{w}}$ is the amount of solids in the wastage. 


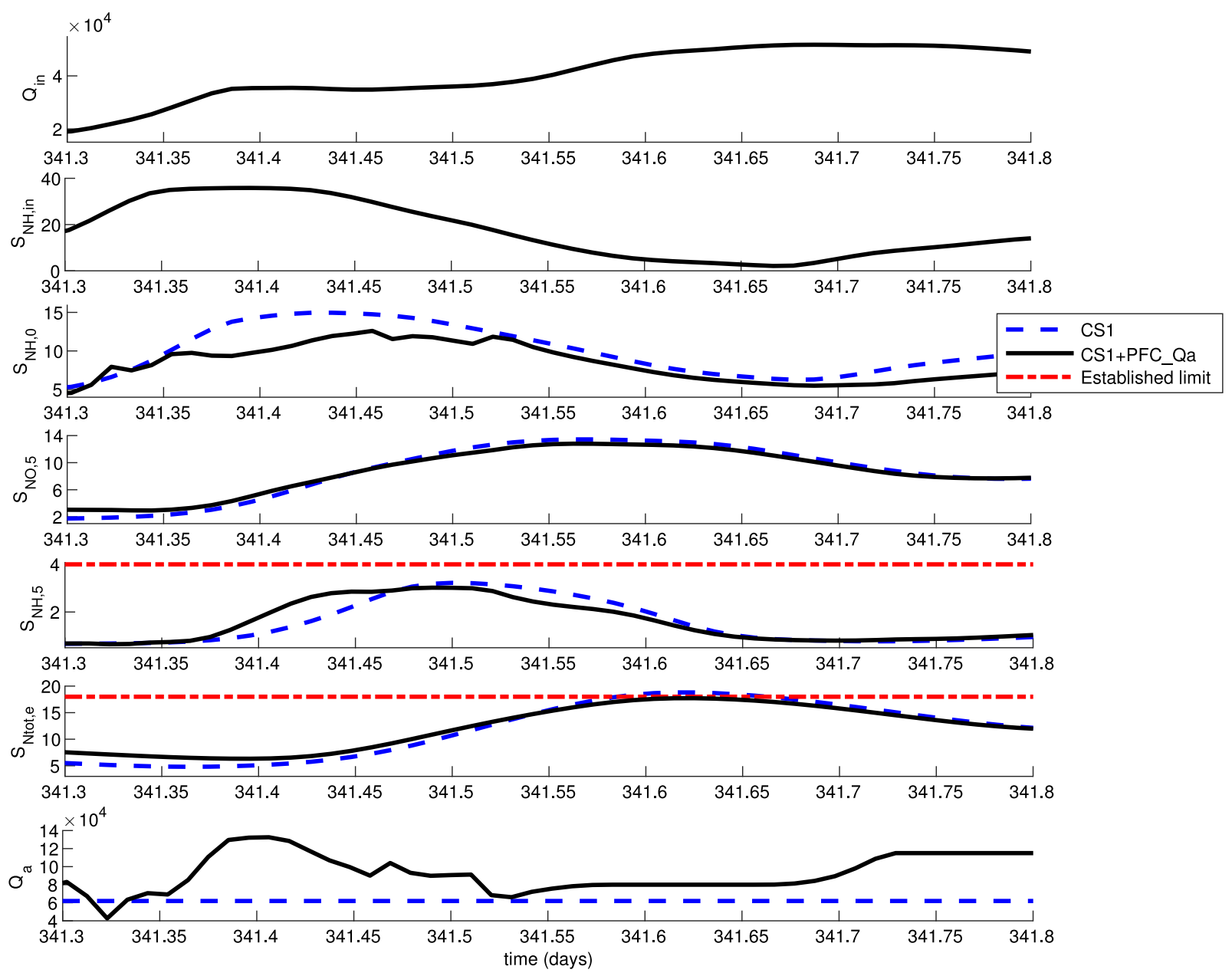

Fig. 5. Time evolution of $Q_{\mathrm{in}}, S_{\mathrm{NH}, \text { in }}, S_{\mathrm{NH}, 0}, S_{\mathrm{NO}, 5}, S_{\mathrm{NH}, 5}, S_{\mathrm{Ntot}, \mathrm{e}}$ and $Q_{\mathrm{a}}$ of day 341 for CS1 and CS1+PFC_Q $Q_{\mathrm{a}}$.

Table A.6

Effluent quality limits.

\begin{tabular}{ll}
\hline Variable & Value \\
\hline $\mathrm{S}_{\mathrm{N}_{\text {tot }}}$ & $<18 \mathrm{~g} \mathrm{~N} \mathrm{~m}^{-3}$ \\
COD & $<100 \mathrm{~g} \mathrm{COD} \mathrm{m}^{-3}$ \\
$\mathrm{~S}_{\mathrm{NH}}$ & $<4 \mathrm{~g} \mathrm{~N} \mathrm{~m}^{-3}$ \\
TSS & $<30 \mathrm{~g} \mathrm{SS} \mathrm{m}^{-3}$ \\
BOD $_{5}$ & $<10 \mathrm{~g} \mathrm{BOD} \mathrm{m}^{-3}$ \\
\hline
\end{tabular}

EC refers to the carbon that could be added to improve denitrification:

$E C=\frac{C O D_{E C}}{T \cdot 1000} \int_{t=245 \text { days }}^{t=609 \text { days }}\left(\sum_{i=1}^{i=n} q_{E C, i}\right) \cdot d t$

where $q_{\mathrm{EC}, \mathrm{i}}$ is the external carbon addition $\left(q_{\mathrm{EC}}\right)$ added to compartment $\mathrm{i}, C O D_{\mathrm{EC}}=400 \mathrm{gCOD} \cdot \mathrm{m}^{-3}$ is the concentration of readily biodegradable substrate in the external carbon source.

$M E$ is a function of the compartment volume and is the energy employed to mix the anoxic tanks to avoid settling (KWh/d):

$M E=\frac{24}{T} \int_{t=245 \text { days }}^{t=609 \text { days }} \sum_{i=1}^{5}\left[0.005 \cdot V_{i}\right.$ if $K_{L} a_{i}(t)<20 d^{-1}$ otherwise 0$] \cdot d t$
$M E T_{\text {prod }}$ is calculated as:

$M E T_{\text {prod }}=\frac{16 \cdot P_{a t m}}{R \cdot T_{a d} \cdot T} \cdot \int_{t=245 \text { days }}^{t=609 \text { days }} \frac{Q_{g a s}(t) \cdot p_{g a s, c h 4}(t)}{P_{\text {gas }}(t)} \cdot d t$

where $P_{\mathrm{atm}}$ is the atmosphere pressure equal to 1.013 bar, $R$ is the gas constant equal to $0.083145 \mathrm{bar} \mathrm{m}^{3} \mathrm{~K}^{-1} \mathrm{kmol}^{-1}, T_{\mathrm{ad}}$ is the temperature in the anaerobic digester, $Q_{\text {gas }}$ is the gas flow rate of the anaerobic digester, $P_{\text {gas }}$ in the gas pressure of the anaerobic digester and $Q p_{\text {gas,ch4 }}$ is the methane pressure of the anaerobic digester.

$H E_{\text {net }}$ is defined as:

$H E_{\text {net }}=\max \left(0 ., H E-7 . \cdot M E T_{\text {prod }}\right)$

where $H E$ is the necessary energy to heat the anaerobic digester to the operating temperature, and it is calculated as:

$H E=\frac{100 \cdot 4186}{86,400 \cdot T} \int_{t=245 \text { days }}^{t=609 \text { days }}\left(T_{a d}-T_{a d, i}\right) \cdot Q_{a d}(t) \cdot d t$

where $T_{\mathrm{ad}, \mathrm{i}}$ is the temperature in the entrance of the anaerobic digester.

The simulation conditions are the ones established in BSM2 by default to fairly compare the results with other works using the same benchmark. Thus, the variable-step solver ode45 is taken. Variable-step size is needed in order to guarantee the best numerical solution as the mathematical model includes components with very different time constants. 


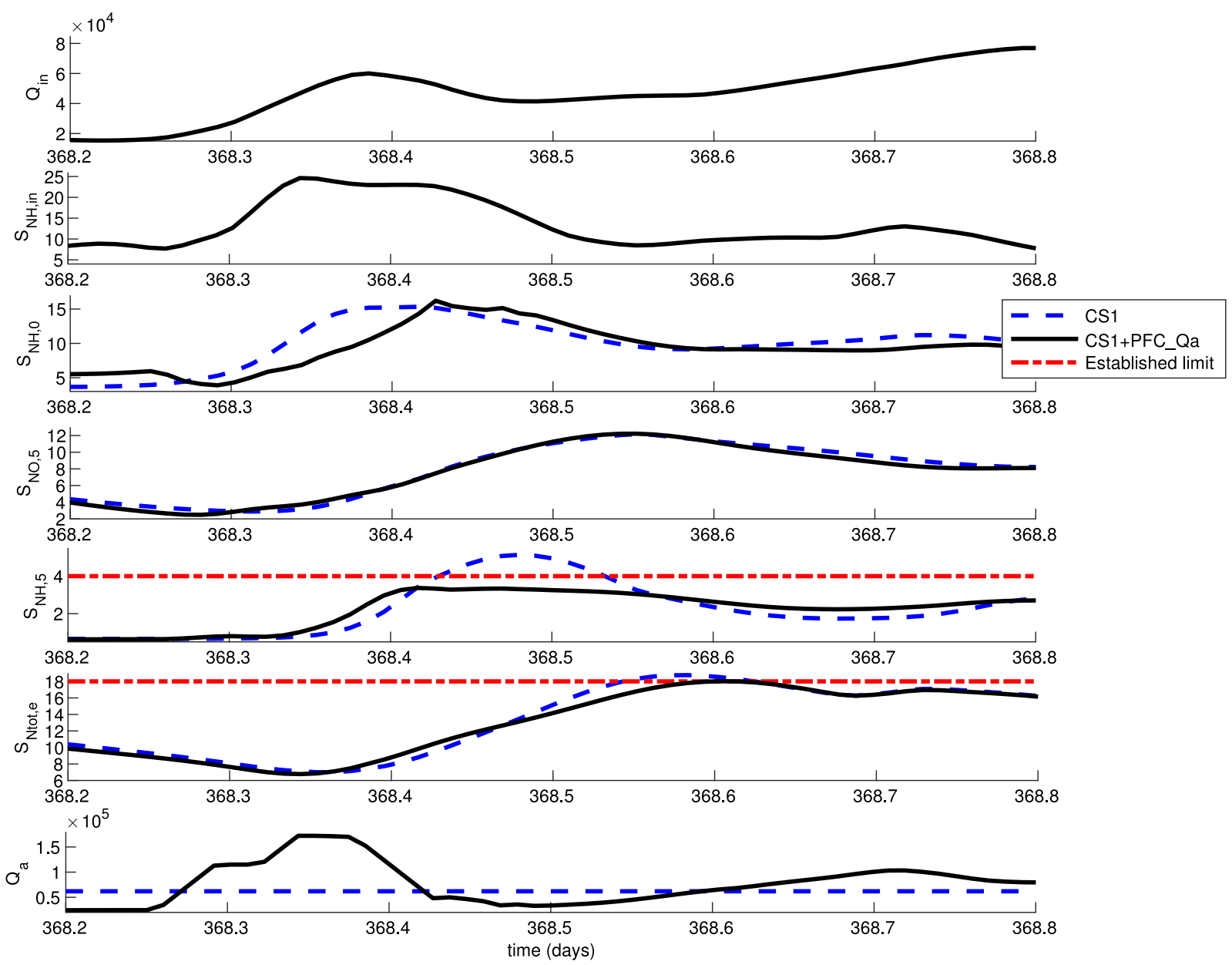

Fig. 6. Time evolution of $Q_{\text {in }}, S_{\mathrm{NH}, \text { in }}, S_{\mathrm{NH}, 0}, S_{\mathrm{NO}, 5}, S_{\mathrm{NH}, 5}, S_{\mathrm{Ntot}, \mathrm{e}}$ and $Q_{a}$ of day 368 for CS1 and CS1+PFC_ $Q_{a}$.

Table A.7

Ranges, types and parameters of the membership functions.

\begin{tabular}{|c|c|c|c|c|c|}
\hline Variables & & Range & Fuzzy sets & Type & Parameters \\
\hline \multirow{6}{*}{ Inputs } & $S_{\mathrm{NH}, \text { in }}$ & [12 40] & $\begin{array}{l}\text { Low } \\
\text { Medium } \\
\text { High }\end{array}$ & $\begin{array}{l}\text { Z-shaped } \\
\text { Triangular } \\
\text { S-shaped }\end{array}$ & 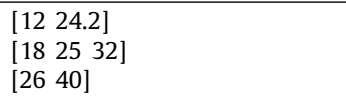 \\
\hline & $S_{\mathrm{NH}, 0}$ & [10 21] & $\begin{array}{l}\text { Low } \\
\text { Medium } \\
\text { High }\end{array}$ & $\begin{array}{l}\text { Z-shaped } \\
\text { Triangular } \\
\text { S-shaped }\end{array}$ & $\begin{array}{l}{\left[\begin{array}{ll}14 & 16.5\end{array}\right]} \\
{\left[\begin{array}{ll}14 & 17.5 \\
{[19} & 21\end{array}\right]}\end{array}$ \\
\hline & $S_{\mathrm{NH}, 5}$ & {$\left[\begin{array}{ll}0.4 & 3.5\end{array}\right]$} & $\begin{array}{l}\text { Low } \\
\text { Medium } \\
\text { High }\end{array}$ & $\begin{array}{l}\text { Z-shaped } \\
\text { Triangular } \\
\text { S-shaped }\end{array}$ & 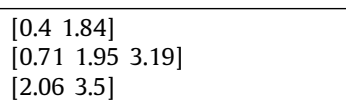 \\
\hline & $S_{\mathrm{NO}, 5}$ & {$\left[\begin{array}{ll}5 & 20\end{array}\right]$} & $\begin{array}{l}\text { Low } \\
\text { Medium } \\
\text { High }\end{array}$ & $\begin{array}{l}\text { Z-shaped } \\
\text { Triangular } \\
\text { S-shaped }\end{array}$ & $\begin{array}{l}{\left[\begin{array}{ll}5 & 11.25\end{array}\right]} \\
{\left[\begin{array}{lll}8.5 & 12 & 15.5\end{array}\right]} \\
{\left[\begin{array}{ll}13 & 20\end{array}\right]}\end{array}$ \\
\hline & $T_{\mathrm{as}}$ & {$\left[\begin{array}{ll}10 & 20\end{array}\right]$} & $\begin{array}{l}\text { Low } \\
\text { High }\end{array}$ & $\begin{array}{l}\text { Z-shaped } \\
\text { S-shaped }\end{array}$ & $\begin{array}{l}{\left[\begin{array}{ll}13 & 17.5\end{array}\right]} \\
{\left[\begin{array}{ll}13.5 & 17\end{array}\right]}\end{array}$ \\
\hline & $Q_{\text {in }}$ & {$[1 \mathrm{e}+045 \mathrm{e}+04]$} & $\begin{array}{l}\text { Low } \\
\text { Medium } \\
\text { High }\end{array}$ & $\begin{array}{l}\text { Z-shaped } \\
\text { Triangular } \\
\text { S-shaped }\end{array}$ & 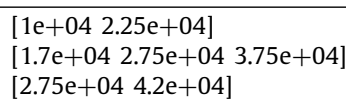 \\
\hline Output & $Q_{a}$ & {$\left[\begin{array}{ll}0 & 2 \mathrm{e}+05\end{array}\right]$} & $\begin{array}{l}\text { Very-low } \\
\text { Low } \\
\text { Medium-Low } \\
\text { Medium } \\
\text { High } \\
\text { Very-High }\end{array}$ & $\begin{array}{l}\text { Triangular } \\
\text { Triangular } \\
\text { Triangular } \\
\text { Triangular } \\
\text { Triangular } \\
\text { Triangular }\end{array}$ & $\begin{array}{l}{\left[\begin{array}{lll}-2.5 \mathrm{e}+04 & 0 & 2.5 \mathrm{e}+04\end{array}\right]} \\
{\left[\begin{array}{llll}5000 & 2.5 \mathrm{e}+04 & 4.5 \mathrm{e}+04\end{array}\right]} \\
{\left[\begin{array}{llll}2.5 \mathrm{e}+04 & 4.54 \mathrm{e}+04 & 6.5 \mathrm{e}+04\end{array}\right]} \\
{\left[\begin{array}{llll}4.5 \mathrm{e}+04 & 8 \mathrm{e}+04 & 1.15 \mathrm{e}+05\end{array}\right]} \\
{\left[\begin{array}{lll}8 \mathrm{e}+04 & 1.15 \mathrm{e}+05 & 1.5 \mathrm{e}+05\end{array}\right]} \\
{\left[\begin{array}{lll}1.15 \mathrm{e}+05 & 2 \mathrm{e}+05 & 2.85 \mathrm{e}+05\end{array}\right]}\end{array}$ \\
\hline
\end{tabular}




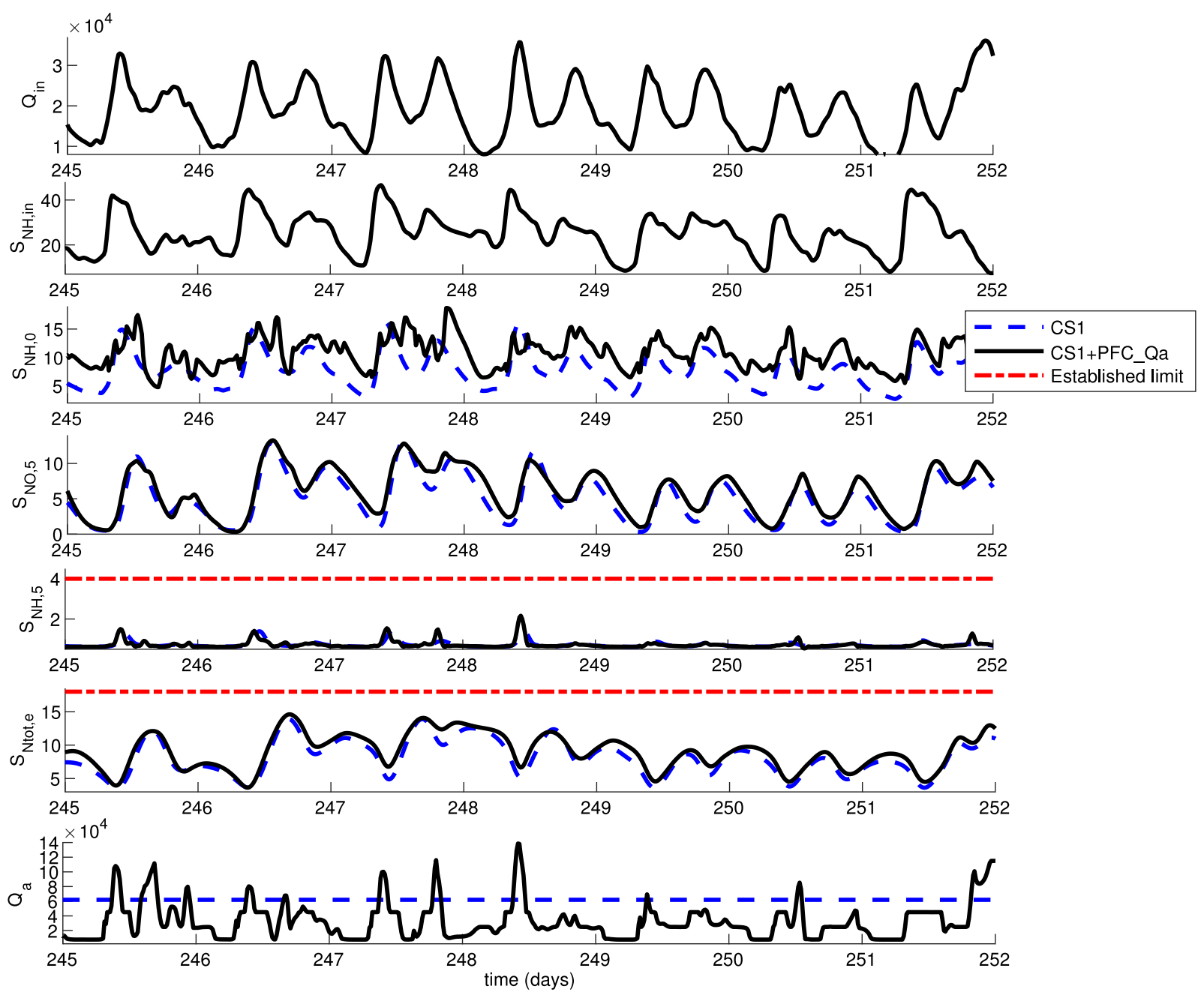

Fig. 7. Time evolution of $Q_{\mathrm{in}}, S_{\mathrm{NH}, \text { in }}, S_{\mathrm{NH}, 0}, S_{\mathrm{NO}, 5}, S_{\mathrm{NH}, 5}, S_{\mathrm{Ntot}, \mathrm{e}}$ and $Q_{\mathrm{a}}$ during one week in summer for CS1 and CS1+PFC_Q $Q_{\mathrm{a}}$.

\section{Control approach}

This article applies a new control strategy to manipulate $Q_{\mathrm{a}}$ by a fuzzy controller, with the aim of reducing limit violations of $S_{\text {Ntot }}$ in the effluent $\left(S_{\mathrm{Ntot}, \mathrm{e}}\right)$ and $S_{\mathrm{NH}}$ in the effluent $\left(S_{\mathrm{NH}, \mathrm{e}}\right)$ as well as operational costs. The idea of the work is to add PFC_ $Q_{a}$ to other already tested and published operation strategies, replacing only the $Q_{a}$ manipulation. The effects of $Q_{a}$ on biological treatment, the design of PFC_ $Q_{a}$ and the operation strategies on which it is tested are detailed below.

\section{1. $Q_{a}$ effects on the biological treatment}

The $Q_{\mathrm{a}}$ variation influences both the denitrification and the nitrification processes, as well as the dilution or increase of concentrations at the biological treatment input. Due to this fact $Q_{a}$ variations have immediate effects on $S_{\mathrm{Ntot}, \mathrm{e}}$ and $S_{\mathrm{NH}, \mathrm{e}}$, but also other different effects on the same variables after some time. This complexity makes necessary an in-depth knowledge of the plant behavior for the $Q_{a}$ manipulation and justify the use of a fuzzy controller.

A more detailed explanation of the $Q_{a}$ influence on the biological treatment, specifically on $S_{\mathrm{Ntot}}$ and $S_{\mathrm{NH}}$, is carried out in Fig. 2 , the equations of the observed conversion rates and mass balance of the biological reactors described in Section 2 and the mixture of concentrations at the inlet of the biological treatment.

\subsection{1. $Q_{\mathrm{a}}$ effect at the inlet of the biological treatment}

On one hand, the $S_{\mathrm{NH}}$ value at the inlet of the first reactor is given by the mixture of $S_{\mathrm{NH}}$ from the primary clarifier $\left(S_{\mathrm{NH}, \mathrm{po}}\right)$ and the recirculated $S_{\mathrm{NH}}$, which is equal to $S_{\mathrm{NH}}$ in the fifth $\operatorname{tank}\left(S_{\mathrm{NH}, 5}\right)$, as can be seen in (21).

$S_{N H, 0}=\frac{Q_{\text {in }} \cdot S_{N H, p o}+Q_{a} \cdot S_{N H, 5}}{Q_{\text {in }}+Q_{a}}$

As due to the nitrification process the $S_{\mathrm{NH}, 5}$ value is lower than $S_{\mathrm{NH}, \text { po }}, Q_{\mathrm{a}}$ increases causes a dilution of $S_{\mathrm{NH}}$ at the input of the first reactor $\left(S_{\mathrm{NH}, 0}\right)$ and, on the contrary, $Q_{a}$ reductions result in $S_{\mathrm{NH}, 0}$ increases. On the other hand, since there is no $S_{\mathrm{NO}}$ in the influent and all $S_{\mathrm{NO}}$ at the inlet of the first tank comes from $Q_{a}$, which is equal to $S_{\mathrm{NO}}$ in the fifth $\operatorname{tank}\left(S_{\mathrm{NO}, 5}\right), Q_{\mathrm{a}}$ increases cause $S_{\mathrm{NO}}$ at the input of the first reactor $\left(S_{\mathrm{NO}, 0}\right)$ increases (22).

$S_{N O, 0}=\frac{Q_{\text {in }} \cdot S_{N O, p o}+Q_{a} \cdot S_{N O, 5}}{Q_{\text {in }}+Q_{a}}$

The $S_{\mathrm{NH}, 0}$ and $S_{\mathrm{NO}, 0}$ values affect $S_{\mathrm{NH}, \mathrm{e}}$ and $S_{\mathrm{Ntot}, \mathrm{e}}$ values after a period of time that depends on HRT and therefore on the flow rate.

\subsection{2. $Q_{a}$ effect on the denitrification process}

In the denitrification process that takes place in anoxic reactors, $S_{\mathrm{NO}}$ is reduced to molecular nitrogen, which is harmless. The active heterotrophic biomass $\left(X_{\mathrm{B}, \mathrm{H}}\right)$ consume $S_{\mathrm{S}}$ using oxygen from $S_{\text {NO }}$ (due to the absence of $S_{O}$ ). Thus, as it can be seen in the 

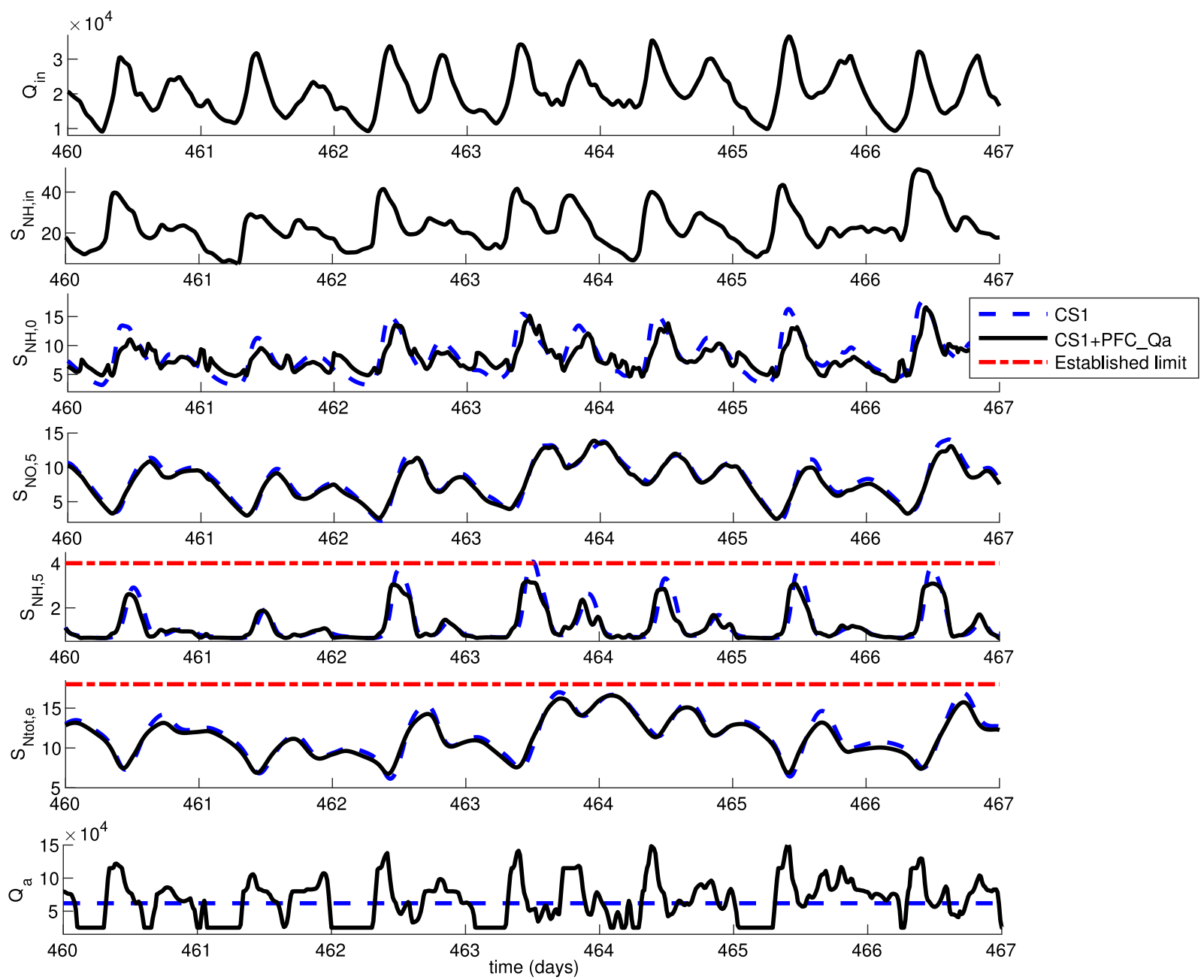

Fig. 8. Time evolution of $Q_{\mathrm{in}}, S_{\mathrm{NH}, \text { in }}, S_{\mathrm{NH}, 0}, S_{\mathrm{NO}, 5}, S_{\mathrm{NH}, 5}, S_{\mathrm{Ntot}, \mathrm{e}}$ and $Q_{\mathrm{a}}$ during one week in winter for CS1 and CS1+PFC_ $Q_{\mathrm{a}}$.

$r_{\mathrm{S}_{\mathrm{NO}}}$ Eqs. (2), (5), the greater the amount of $S_{\mathrm{S}}$, the greater the $S_{\mathrm{NO}}$ reduction. Due to the fact that $S_{S}$ is reduced during the biological treatment, an increase in $Q_{a}$ reduces $S_{N O}$ in the anoxic reactors, worsening denitrification.

A more important effect of $Q_{a}$ on the denitrification process is its influence on HRT, since when the flow is higher, HRT decreases and vice versa. As can been observed in the mass balance equation (11), an increase in $Q_{a}$ diminishes the effect of $r_{Z}$ and therefore worsens the denitrification process, increasing $S_{\mathrm{NO}}$ and consequently $S_{\text {Ntot,e }}$.

\subsection{3. $Q_{a}$ effect on the nitrification process}

The nitrification process takes place in aerobic reactors, where the active autotrophic biomass $\left(X_{\mathrm{B}, \mathrm{A}}\right)$ oxidize $S_{\mathrm{NH}}$ into $S_{\mathrm{NO}}$. In the same way as in the denitrification process, $Q_{\mathrm{a}}$ increases attenuate the effect of $r_{Z}$ (11), worsening in this case the nitrification process, which causes $S_{\mathrm{NH}}$ increase and $S_{\mathrm{NO}}$ decrease. However, $S_{\mathrm{O}}$ is often regulated in the aerobic reactors based on $S_{\mathrm{NH}}$. In this case, $Q_{a}$ increases can result in an $S_{0}$ increase to improve the nitrification process, which reduces $S_{\mathrm{NH}}$ and increases $S_{\mathrm{NO}}$. In conclusion, the best way to reduce $S_{\mathrm{NH}}$ is to reduce $Q_{\mathrm{a}}$. Regarding $S_{\mathrm{Ntot}}$, as its main components are $S_{\mathrm{NH}}$ and $S_{\mathrm{NO}}$, a trade-off solution must be found between both to reduce $S_{\mathrm{Ntot}}$.

\subsection{Fuzzy controller design}

Fuzzy logic can be defined as a control based on human expertise. Fuzzy controller adapts the input and output variables into suitable linguistic values by membership functions. Rules between input and output variables are established by words. Non-expert readers can found further information about fuzzy control in standard references such as Klir and Yuan [31]. The FIS ${ }^{2}$ Editor from Matlab is used in this work for the implementation of PFC_ $Q_{a}$.

As explained in Section 3.1, to assess the $Q_{a}$ effects on the biological treatment requires an exhaustive knowledge of the plant behavior. Due to this reason, a fuzzy controller has been proposed for the $Q_{a}$ manipulation.

PFC_ $Q_{a}$ has been initially tuned based on the knowledge of the biological processes described by the extended ASM1 and on a specific analysis of the evolution over time of the fuzzy controller inputs. After that, the range of values of the membership functions have been adjusted by trial and error to optimize the results. PFC_ $Q_{\mathrm{a}}$ is conceived here with 6 inputs, 1 output and 30 rules. As shown in Fig. 4, the inputs are $S_{\mathrm{NH}}$ in the influent $\left(S_{\mathrm{NH}, \text { in }}\right), S_{\mathrm{NH}, 0}, S_{\mathrm{NH}, 5}, S_{\mathrm{NO}, 5}, T_{\text {as }}$ and influent flow rate $\left(Q_{\text {in }}\right)$ and the output is $Q_{a}$. Mamdani (Mamdani [32]) is the method of inference

2 FIS: Fuzzy Inference System. 


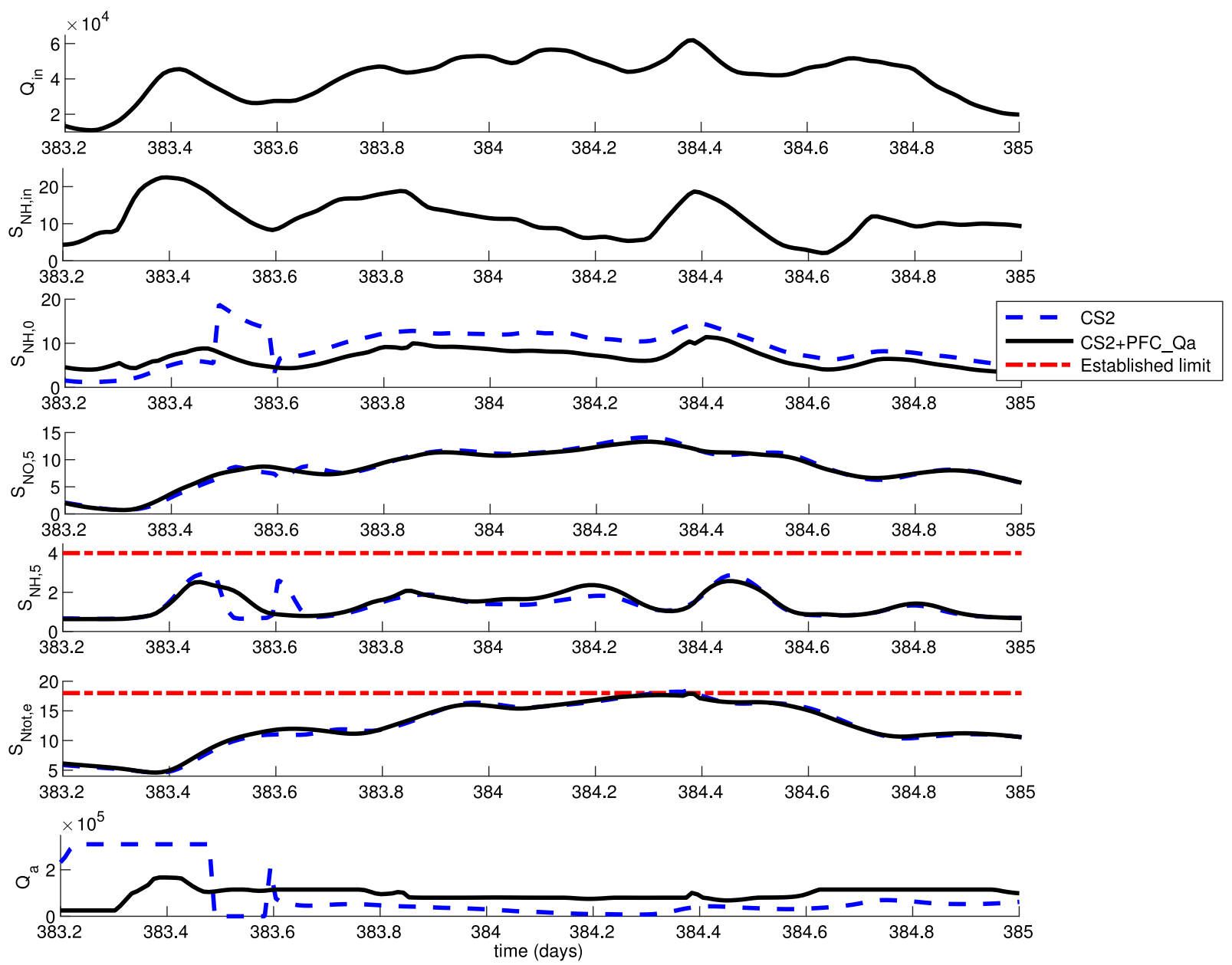

Fig. 9. Time evolution of $Q_{i n}, S_{\mathrm{NH}, \text { in }}, S_{\mathrm{NH}, 0}, S_{\mathrm{NO}, 5}, S_{\mathrm{NH}, 5}, S_{\mathrm{Ntot}, \mathrm{e}}$ and $Q_{a}$ of day 384 for CS2 and CS2+PFC_$Q_{a}$.

Table A.8

Rules of PFC_ $Q_{a}$.

\begin{tabular}{|c|c|c|c|c|c|c|c|}
\hline Rule number & $S_{\mathrm{NH}, \text { in }}$ & $S_{\mathrm{NH}, 0}$ & $S_{\mathrm{NH}, 5}$ & $S_{\mathrm{NO}, 5}$ & $\mathrm{~T}_{\mathrm{as}}$ & $Q_{\text {in }}$ & $Q_{a}$ \\
\hline 1 & If is Low & and is not High & - & - & and is Low & and is Low & Then is Low \\
\hline 2 & If is Low & and is not High & - & - & and is High & and is Low & Then is Very-Low \\
\hline 3 & If is Low & - & and is not High & and is Low & - & and is Medium & Then is High \\
\hline 4 & If is Low & - & and is not High & and is Low & - & and is High & Then is High \\
\hline 5 & If is Low & - & and is not High & and is Medium & - & and is Medium & Then is Medium \\
\hline 6 & If is Low & - & and is not High & and is Medium & - & and is High & Then is Medium \\
\hline 7 & If is Low & - & and is not High & and is High & and is High & - & Then is Very-Low \\
\hline 8 & If is Low & - & and is not High & and is High & and is Low & - & Then is Low \\
\hline 9 & If is Medium & and is not High & and is not High & and is Low & and is High & and is not High & Then is Low \\
\hline 10 & If is Medium & and is not High & and is not High & and is Medium & and is High & and is not High & Then is Very-Low \\
\hline 11 & If is Medium & - & and is not High & and is High & and is High & and is not High & Then is Very-Low \\
\hline 12 & If is Medium & and is Low & and is not High & and is Low & and is Low & and is not High & Then is Medium \\
\hline 13 & If is Medium & and is Low & and is not High & and is Medium & and is Low & and is not High & Then is Medium-Low \\
\hline 14 & If is Medium & - & and is not High & and is High & and is Low & and is not High & Then is Low \\
\hline 15 & If is Medium & - & and is not High & and is Low & - & and is High & Then is Very-High \\
\hline 16 & If is Medium & - & and is not High & and is Medium & - & and is High & Then is Very-High \\
\hline 17 & If is Medium & - & and is not High & and is High & - & and is High & Then is Medium-Low \\
\hline 18 & If is High & and is not High & and is not High & and is Low & and is High & and is not High & Then is Medium-Low \\
\hline 19 & If is High & and is not High & and is not High & and is Medium & and is High & and is not High & Then is Medium-Low \\
\hline 20 & If is High & - & and is not High & and is High & and is High & and is not High & Then is Very-Low \\
\hline 21 & If is High & and is Low & and is not High & and is Low & and is Low & and is not High & Then is High \\
\hline 22 & If is High & and is Low & and is not High & and is Medium & and is Low & and is not High & Then is High \\
\hline 23 & If is High & - & and is not High & and is High & and is Low & and is not High & Then is Medium-Low \\
\hline 24 & If is High & - & and is not High & and is Low & - & and is High & Then is Very-High \\
\hline 25 & If is High & - & and is not High & and is Medium & - & and is High & Then is Very-High \\
\hline 26 & If is High & - & and is not High & and is High & - & and is High & Then is Medium-Low \\
\hline 27 & - & - & If is High & - & and is Low & - & Then is Low \\
\hline 28 & - & - & If is High & - & and is High & - & Then is Very-Low \\
\hline 29 & - & If is not Low & and is not High & and is not High & and is Low & and is not High & Then is High \\
\hline 30 & - & If is High & and is not High & and is not High & and is High & and is not High & Then is High \\
\hline
\end{tabular}




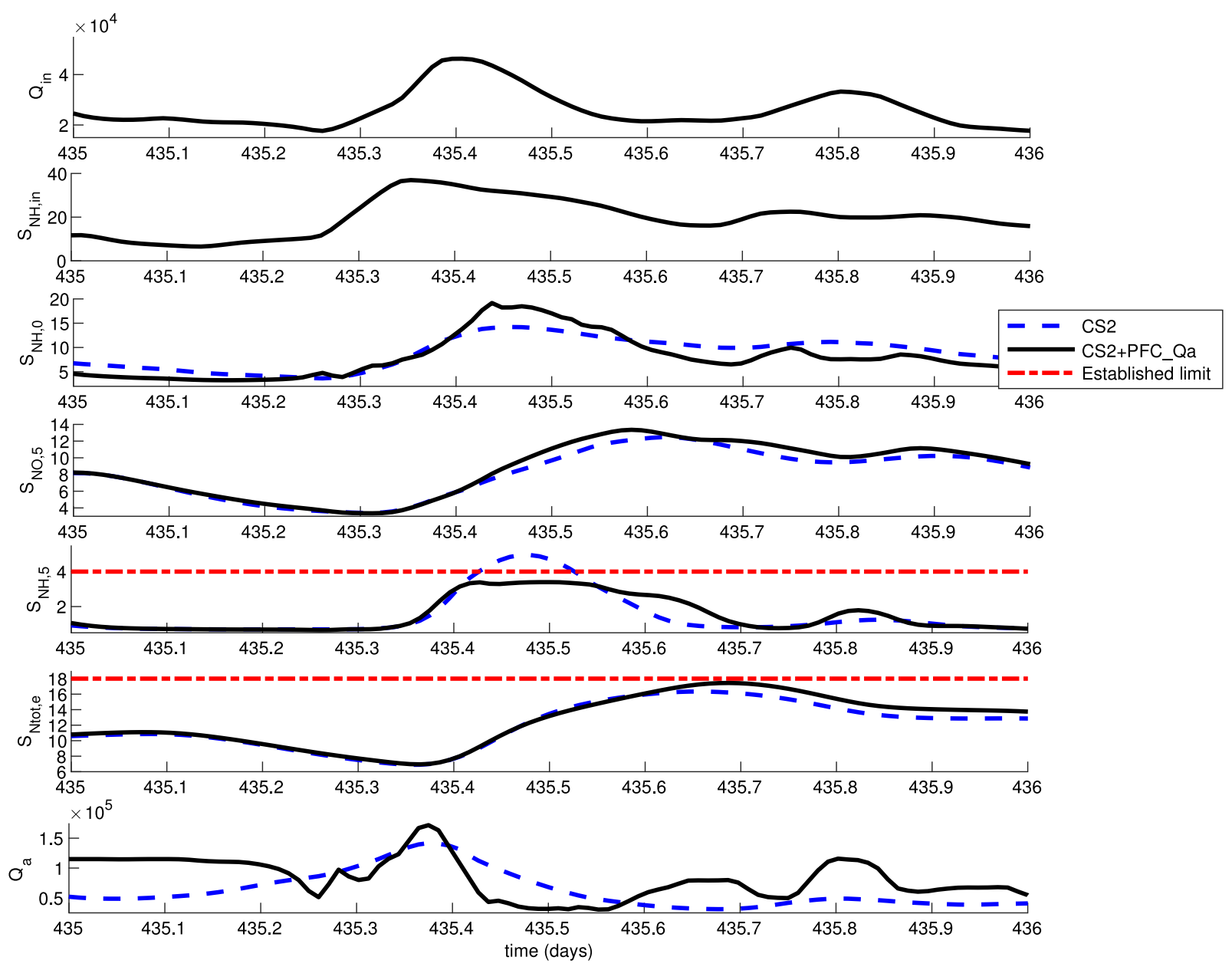

Fig. 10. Time evolution of $Q_{\text {in }}, S_{\mathrm{NH}, \text { in }}, S_{\mathrm{NH}, 0}, S_{\mathrm{NO}, 5}, S_{\mathrm{NH}, 5}, S_{\mathrm{Ntot}, \mathrm{e}}$ and $Q_{a}$ of day 435 for CS2 and CS2+PFC_ $Q_{\mathrm{a}}$.

Table A.9

Parameters of PFC $\_Q_{a}$ that affects the computational complexity.

Number of inputs Number of input fuzzy sets Number of rules Number of output fuzzy sets

\begin{tabular}{llll}
\hline 6 & 17 & 30 & 6
\end{tabular}

Table A.10

CPU time and elapsed time in the 609-day simulation of all the operation strategies with and without PFC $Q_{\mathrm{a}}$.

\begin{tabular}{|c|c|c|c|c|c|c|c|c|c|c|c|c|c|c|c|}
\hline Indicators & CS1 & CS1+PFC_Qa & Difference & CS2 & CS2+PFC_Qa & Difference & CS3 & CS3+PFC_Qa & Difference & CS4 & CS4+PFC_Qa & Difference & CS5 & CS5+PFC_Qa & Difference \\
\hline $\begin{array}{l}\text { CPU time (hours) } \\
\text { Elapsed time (hours) }\end{array}$ & $\begin{array}{l}1.218 \\
1.214\end{array}$ & $\begin{array}{l}1.529 \\
1.512\end{array}$ & $\begin{array}{l}25.534 \% \\
24.547 \%\end{array}$ & $\begin{array}{l}10.239 \\
10.095\end{array}$ & $\begin{array}{l}9.372 \\
9.175\end{array}$ & $\begin{array}{l}-8.467 \% \\
-9.117 \%\end{array}$ & $\begin{array}{l}7.982 \\
7.945\end{array}$ & $\begin{array}{l}7.591 \\
7.469\end{array}$ & $\begin{array}{l}-4.898 \% \\
-5.982 \%\end{array}$ & $\begin{array}{l}7.819 \\
7.671\end{array}$ & $\begin{array}{l}8.074 \\
7.924\end{array}$ & $\begin{array}{l}3.258 \% \\
3.291 \%\end{array}$ & $\begin{array}{l}8.084 \\
7.955\end{array}$ & $\begin{array}{l}7.911 \\
7.757\end{array}$ & $\begin{array}{l}-2.131 \% \\
-2.490 \%\end{array}$ \\
\hline
\end{tabular}

used in this paper. The range, parameter values and types of all the membership functions of the inputs and output of the fuzzy controller are shown in Table A.7 and the rules in Table A.8. In addition the fuzzy controller code is listed in the appendix.

In the Simulink model, $Q_{a}$ variations are limited to a rising and falling slew rates of 2,500,000 and $-2,500,000$ respectively, with flow rate units in $\mathrm{m}^{3} / \mathrm{s}$ and time units in days.

The sample time of the controller applied in a real scenario could take any fixed value that was considered the most suitable to meet the plants' requirements. The lowest possible discretization could also be adopted, but other requirements might make larger values convenient. Applying a sample time based on the maximum response time of the sensors could be an option. Following the BSM2 recommendations on the type of sensors to be applied, $Q_{\text {in }}$ and $T_{\text {as }}$ sensors have a one-minute response time, while $S_{\mathrm{NH}}$ and $S_{\mathrm{NO}}$ sensors have a 10 -minute response time. Thus, an option in a real scenario could be to apply a sample time of 10 min (0.0069 days).
The rules are based on the effects of $Q_{a}$ on the biological treatment explained in Section 3.1 and are detailed below. Some of them are also shown in the surface graphs (Fig. 3).

In dry weather, the ratio of $Q_{\text {in }}$ to $S_{\mathrm{NH}}$, in remains stable, and consequently when there is an increase in this ratio due to an increase in $Q_{\text {in }}$ it is considered that a rain event is taking place. During dry weather, when $S_{\mathrm{NH} \text {, in }}$ is low, $Q_{\mathrm{a}}$ is decreased to reduce pumping energy costs and improve nitrification and denitrification processes, and if $S_{\mathrm{NH} \text {,in }}$ increases, $Q_{\mathrm{a}}$ is also increased in order to dilute $S_{\mathrm{NH}, 0}$. In the case that a rain event is detected, $Q_{\mathrm{a}}$ is increased to dilute $S_{\mathrm{NH}, 0}$, as long as there is no risk of $S_{\mathrm{NH}, 5}$ or $S_{\mathrm{NO}, 5}$ increase. Fig. 3a shows the relationship between $Q_{\text {in }}$ and $S_{\mathrm{NH} \text {,in }}$.

During both dry and rainy weather (except when $S_{\mathrm{NH} \text {, in }}$ is low in dry weather), $Q_{a}$ values are inversely based on the $S_{\mathrm{NO}, 5}$ values, so the higher $S_{\mathrm{NO}, 5}$, the lower $Q_{\mathrm{a}}$ (Fig. $3 \mathrm{~b}$ ). This is because PFC_ $Q_{\mathrm{a}}$ is applied with control strategies that regulate $S_{O}$ set-point based on $S_{\mathrm{NH}}$, since they give better results than keeping $S_{\mathrm{O}}$ set-point fixed (Santín et al. [14]). So, decreasing $Q_{a}, S_{\mathrm{NH}, 5}$ is reduced and 


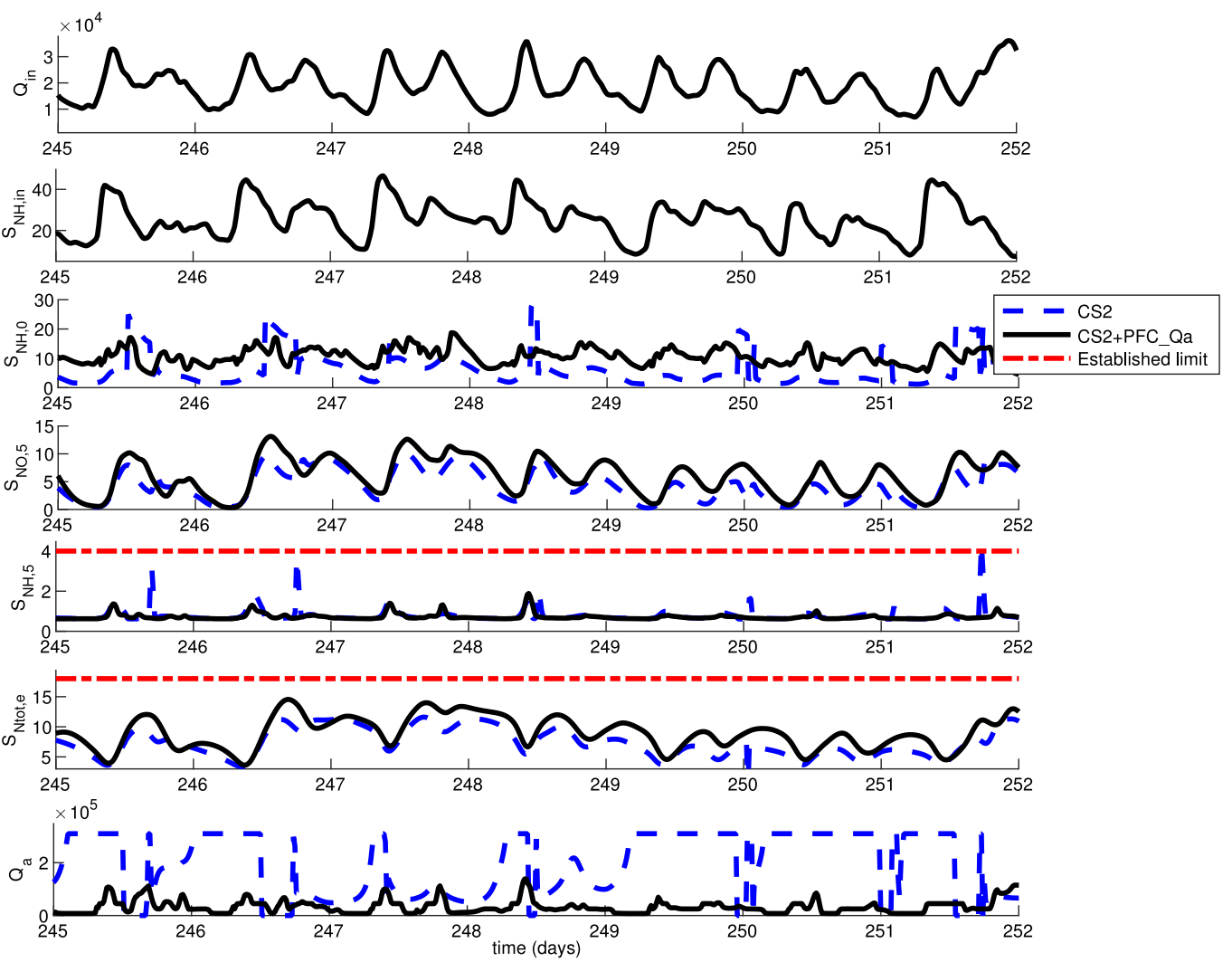

Fig. 11. Time evolution of $Q_{\text {in }}, S_{\mathrm{NH}, \text { in }}, S_{\mathrm{NH}, 0}, S_{\mathrm{NO}, 5}, S_{\mathrm{NH}, 5}, S_{\mathrm{Ntot}, \mathrm{e}}$ and $Q_{\mathrm{a}}$ during one week in summer for CS2 and CS2+PFC_Q

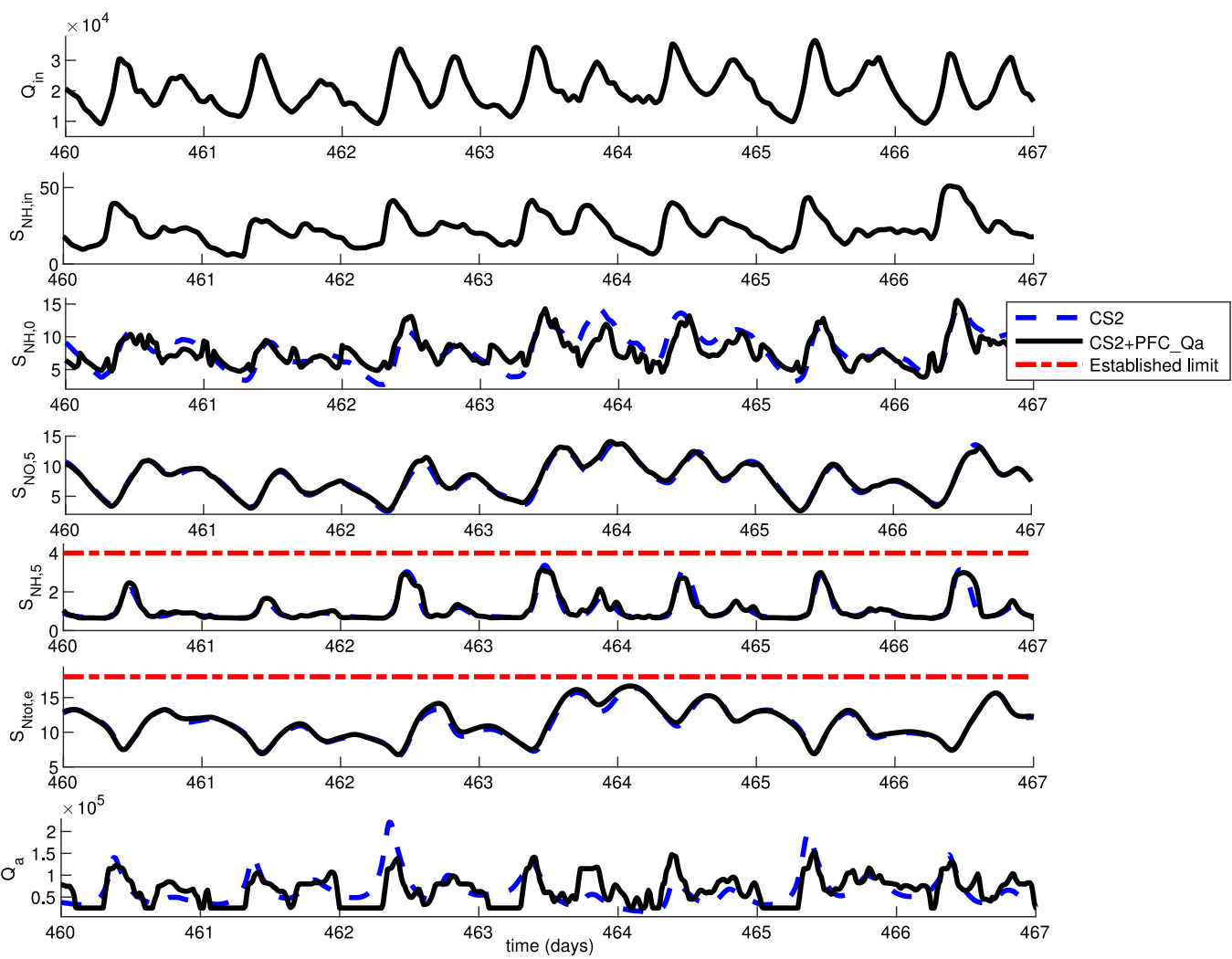

Fig. 12. Time evolution of $Q_{\text {in }}, S_{\mathrm{NH}, \text { in }}, S_{\mathrm{NH}, 0}, S_{\mathrm{NO}, 5}, S_{\mathrm{NH}, 5}, S_{\mathrm{Ntot}, \mathrm{e}}$ and $Q_{\mathrm{a}}$ during one week in winter for CS2 and CS2+PFC_ $Q_{\mathrm{a}}$.

consequently also $S_{\mathrm{O}}$ and $S_{\mathrm{NO}, 0}$. It should be noted that this fact does not happen in the case that $S_{\mathrm{NH}, 5}$ is so high that the value of $S_{0,5}$ reaches its maximum value defined by the controller that regulates it, even if $Q_{a}$ is reduced. In the case of control strategies 


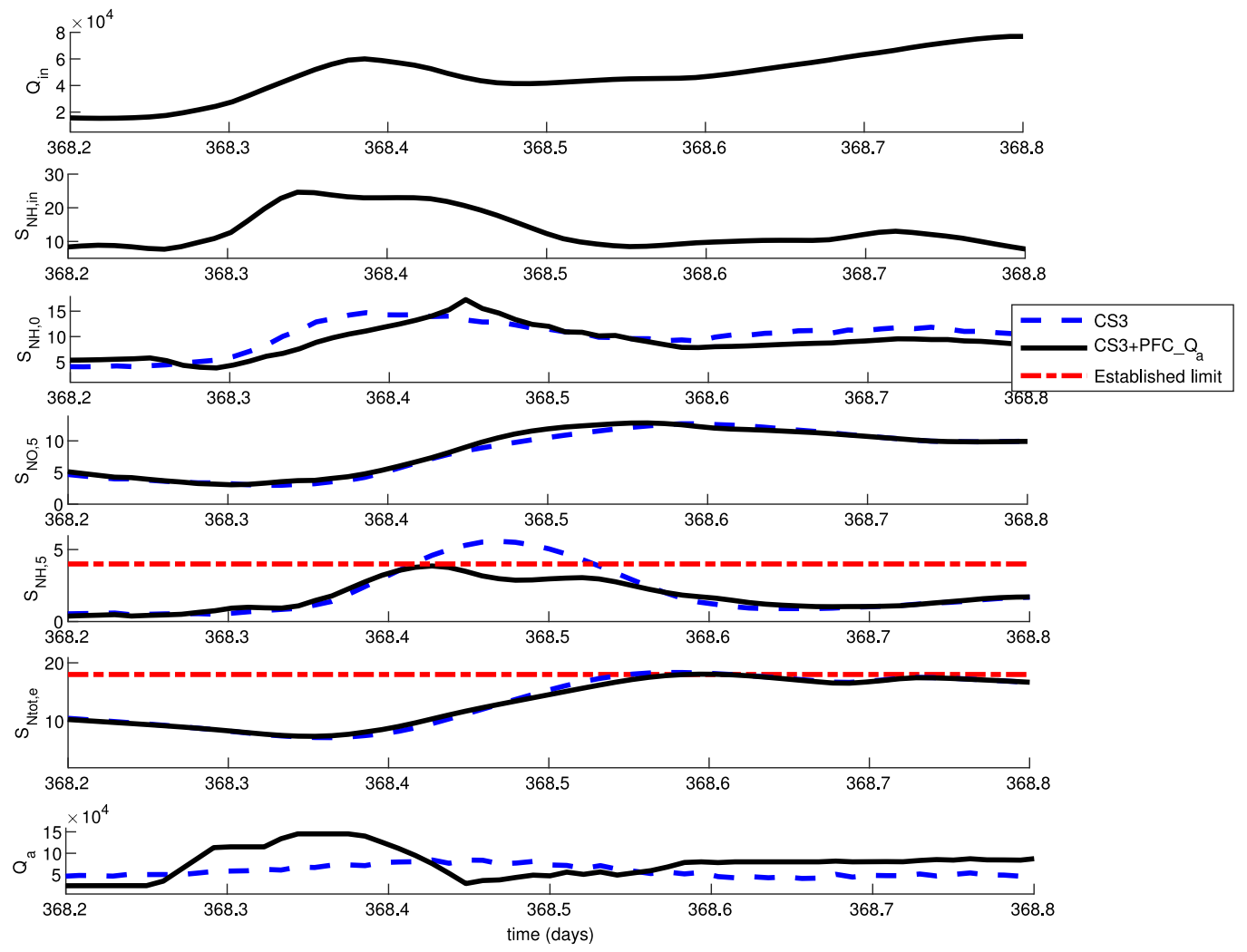

Fig. 13. Time evolution of $Q_{\mathrm{in}}, S_{\mathrm{NH}, \text { in }}, S_{\mathrm{NH}, 0}, S_{\mathrm{NO}, 5}, S_{\mathrm{NH}, 5}, S_{\mathrm{Ntot}, \mathrm{e}}$ and $Q_{\mathrm{a}}$ of day 368 for CS3 and CS3+PFC_ $Q_{\mathrm{a}}$.
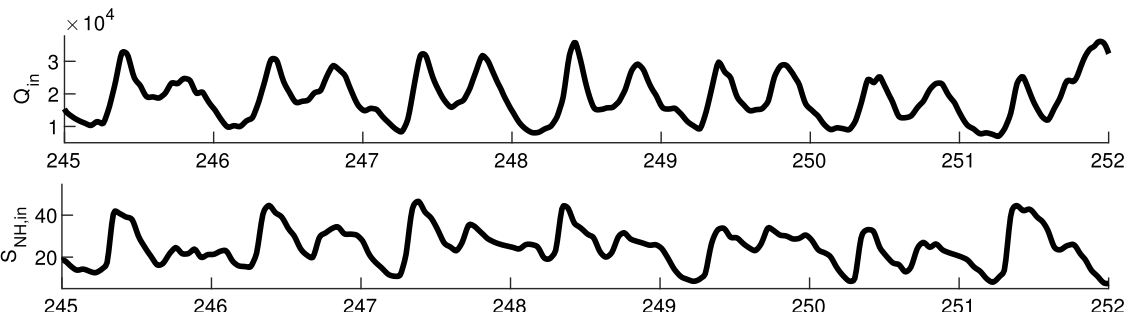

245
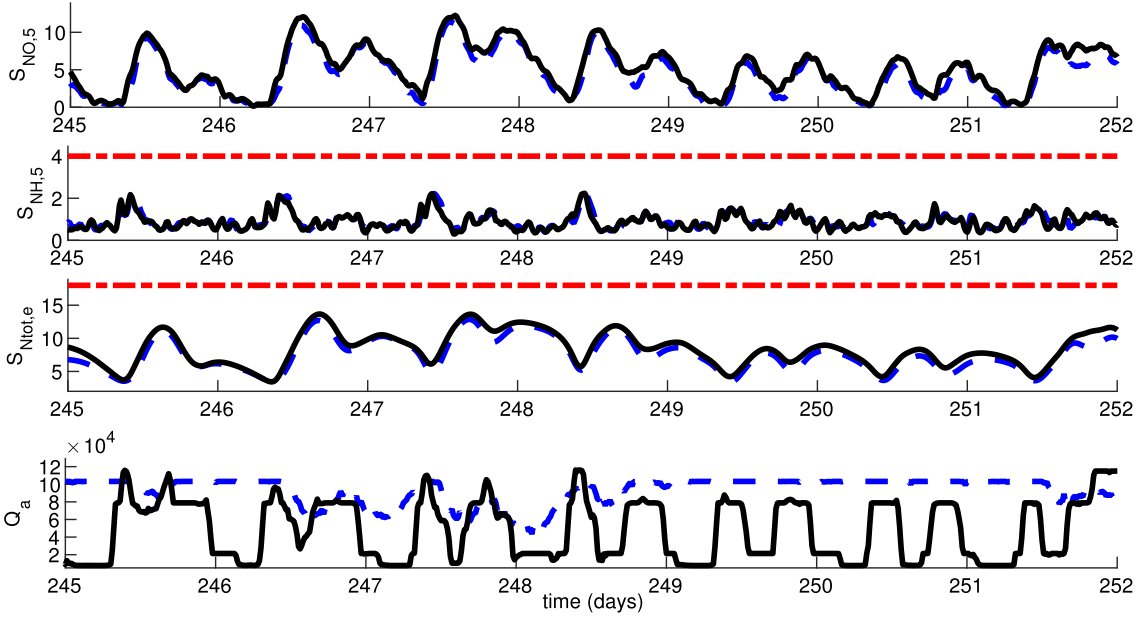

Fig. 14. Time evolution of $Q_{\mathrm{in}}, S_{\mathrm{NH}, \text { in }}, S_{\mathrm{NH}, 0}, S_{\mathrm{NO}, 5}, S_{\mathrm{NH}, 5}, S_{\mathrm{Ntot}, \mathrm{e}}$ and $Q_{\mathrm{a}}$ during one week in summer for CS3 and CS3+PFC_ $Q_{a}$. 


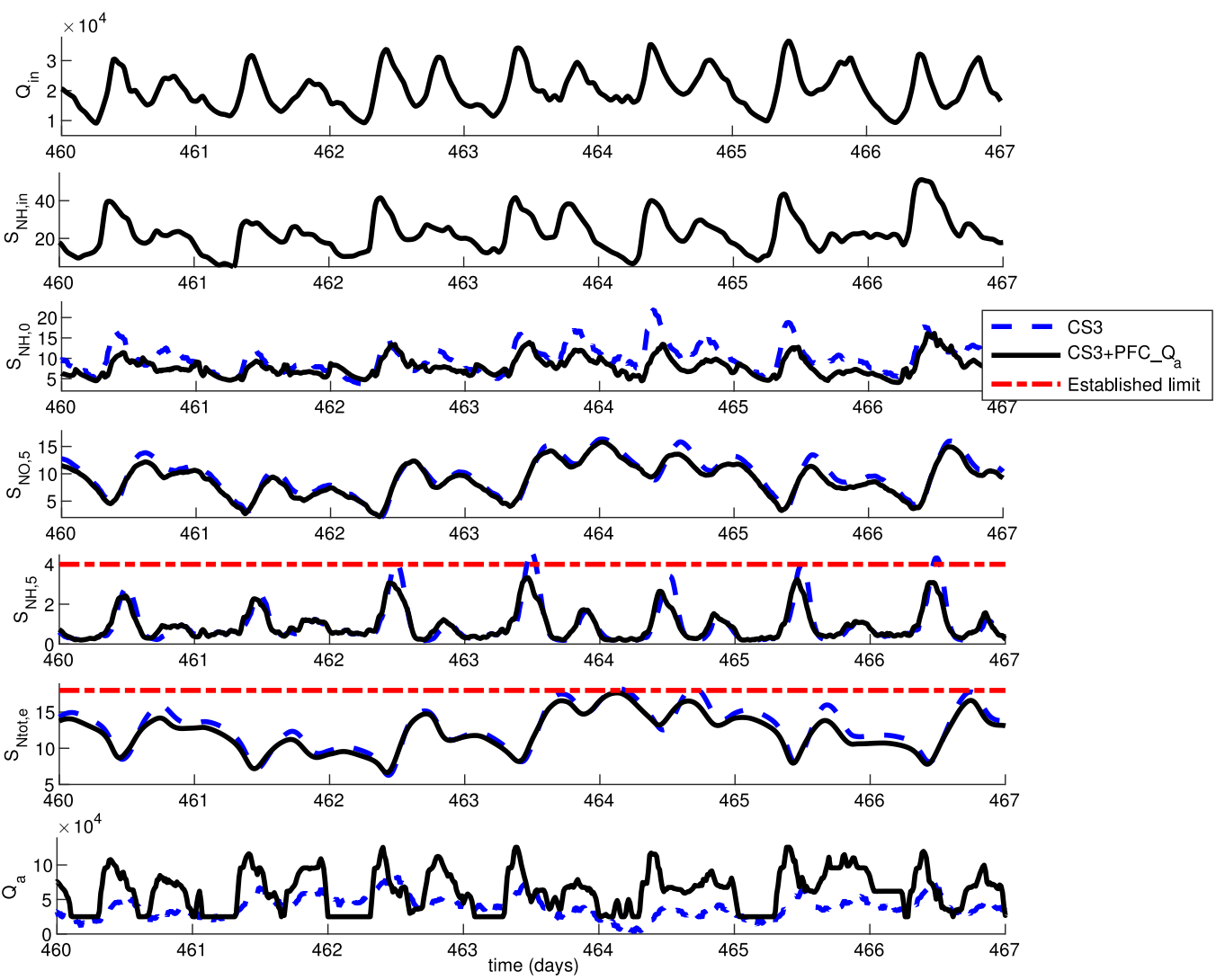

Fig. 15. Time evolution of $Q_{\text {in }}, S_{\mathrm{NH}, \text { in }}, S_{\mathrm{NH}, 0}, S_{\mathrm{NO}, 5}, S_{\mathrm{NH}, 5}, S_{\mathrm{Ntot}, \mathrm{e}}$ and $Q_{\mathrm{a}}$ during one week in winter for CS3 and CS3+PFC_Q $Q_{\mathrm{a}}$.
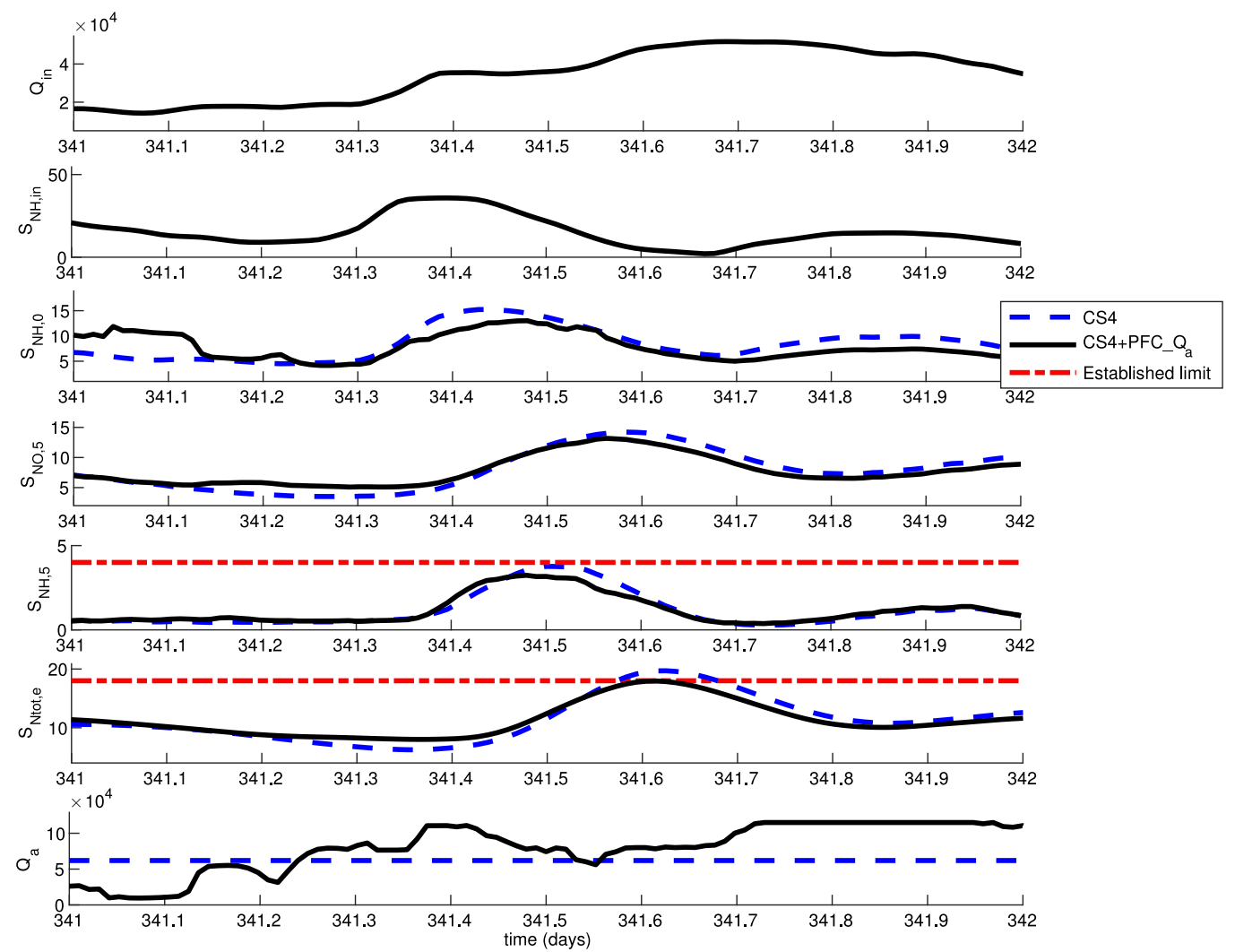

Fig. 16. Time evolution of $Q_{\text {in }}, S_{\mathrm{NH}, \text { in }}, S_{\mathrm{NH}, 0}, S_{\mathrm{NO}, 5}, S_{\mathrm{NH}, 5}, S_{\mathrm{Ntot}, \mathrm{e}}$ and $Q_{\mathrm{a}}$ of day 341 for CS4 and CS4+PFC_ $Q_{\mathrm{a}}$. 


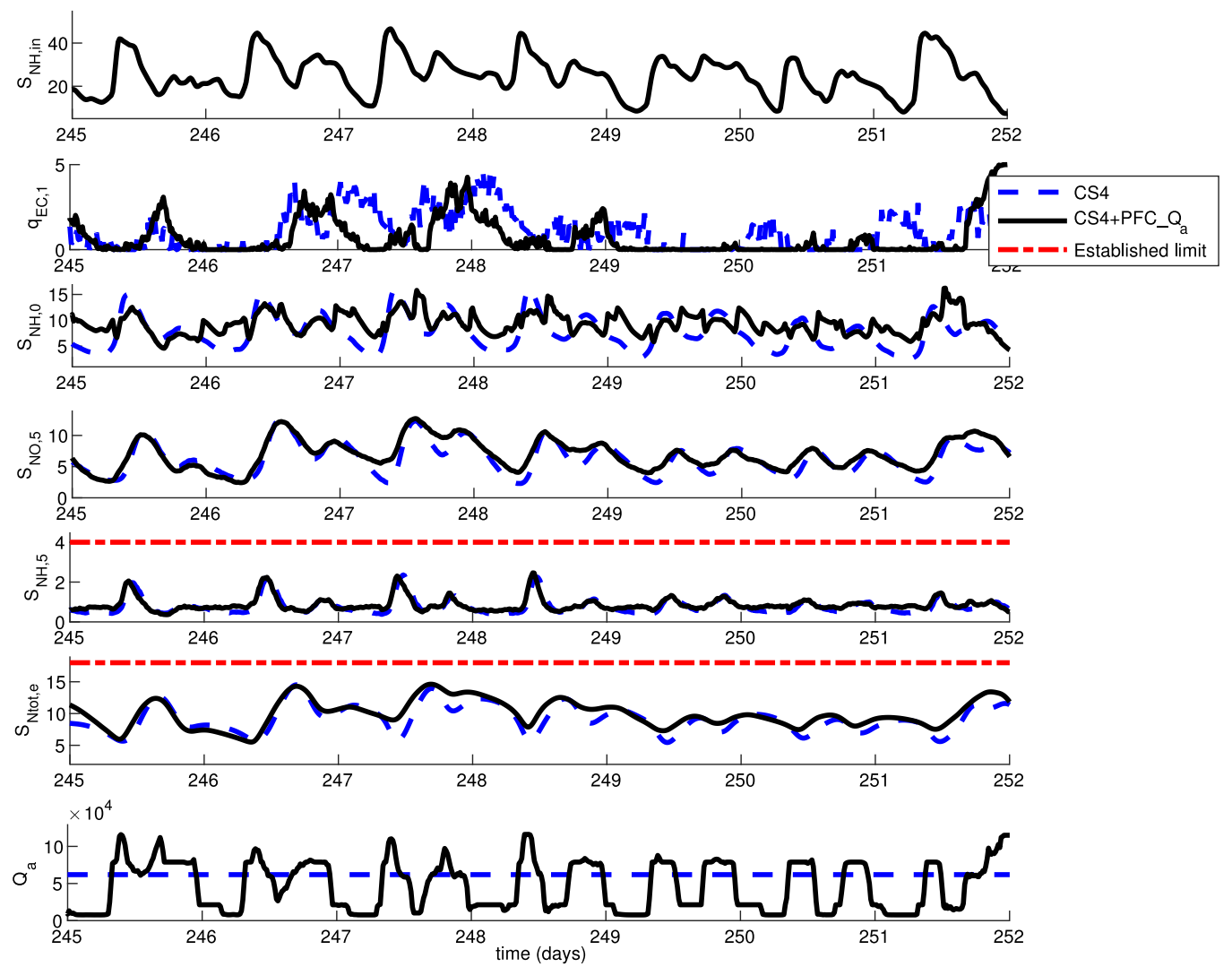

Fig. 17. Time evolution of $S_{\mathrm{NH}, \text { in }}, q_{\mathrm{EC}, 1}, S_{\mathrm{NH}, 0}, S_{\mathrm{NO}, 5}, S_{\mathrm{NH}, 5}, S_{\mathrm{Ntot}, e}$ and $Q_{\mathrm{a}}$ during one week in summer for CS4 and CS4+PFC_ $Q_{\mathrm{a}}$.
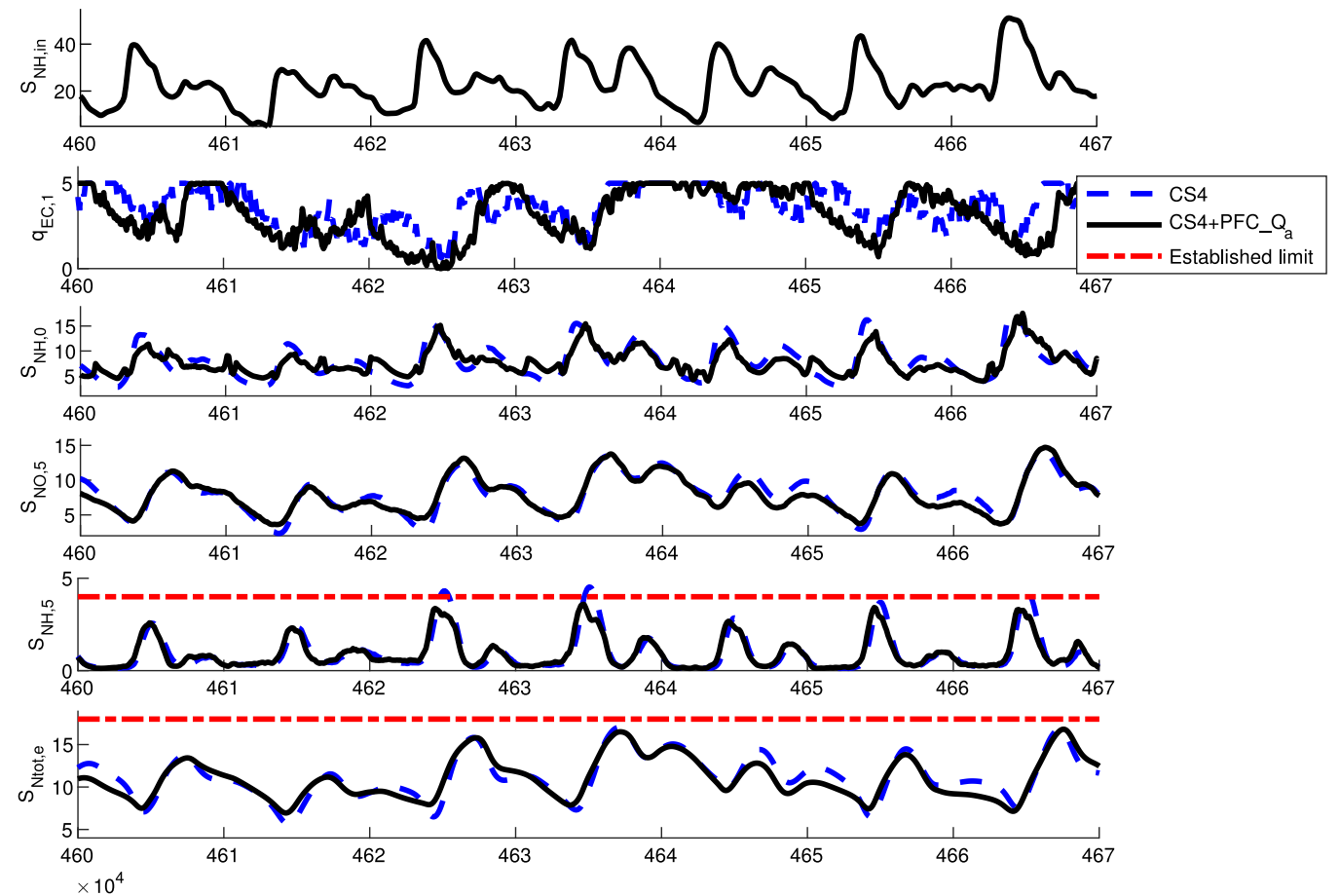

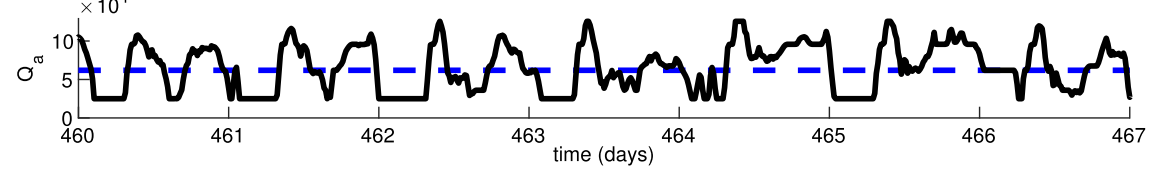

Fig. 18. Time evolution of $S_{\mathrm{NH}, \text { in }}, q_{\mathrm{EC}, 1}, S_{\mathrm{NH}, 0}, S_{\mathrm{NO}, 5}, S_{\mathrm{NH}, 5}, S_{\mathrm{Ntot}, \mathrm{e}}$ and $Q_{\mathrm{a}}$ during one week in winter for CS4 and CS4+PFC_Q $Q_{\mathrm{a}}$. 

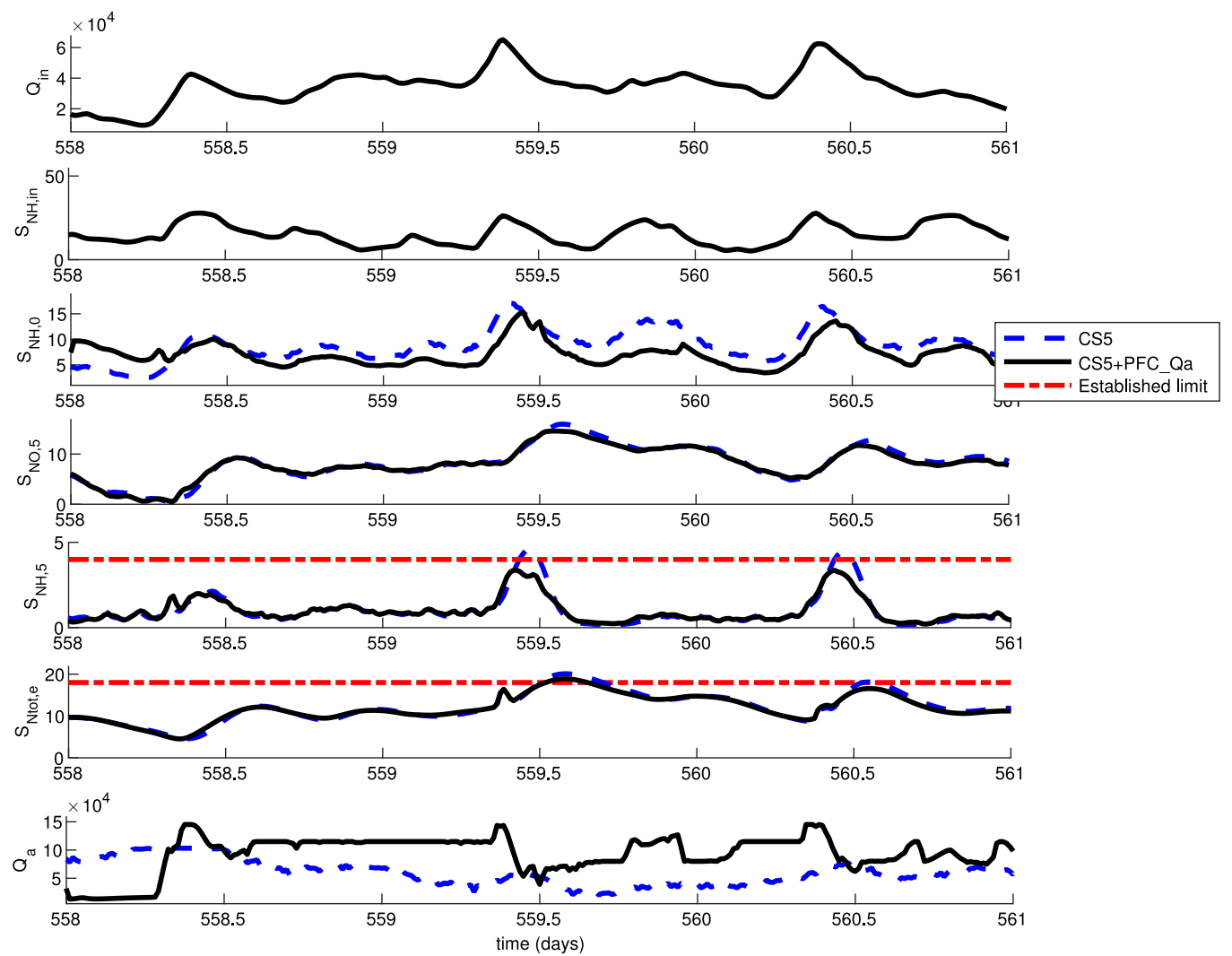

Fig. 19. Time evolution of $Q_{i n}, S_{\mathrm{NH}, \text { in }}, S_{\mathrm{NH}, 0}, S_{\mathrm{NO}, 5}, S_{\mathrm{NH}, 5}, S_{\mathrm{Ntot}, \mathrm{e}}$ and $Q_{a}$ of days 558,559 and 560 for CS5 and CS5+PFC_ $Q_{a}$.
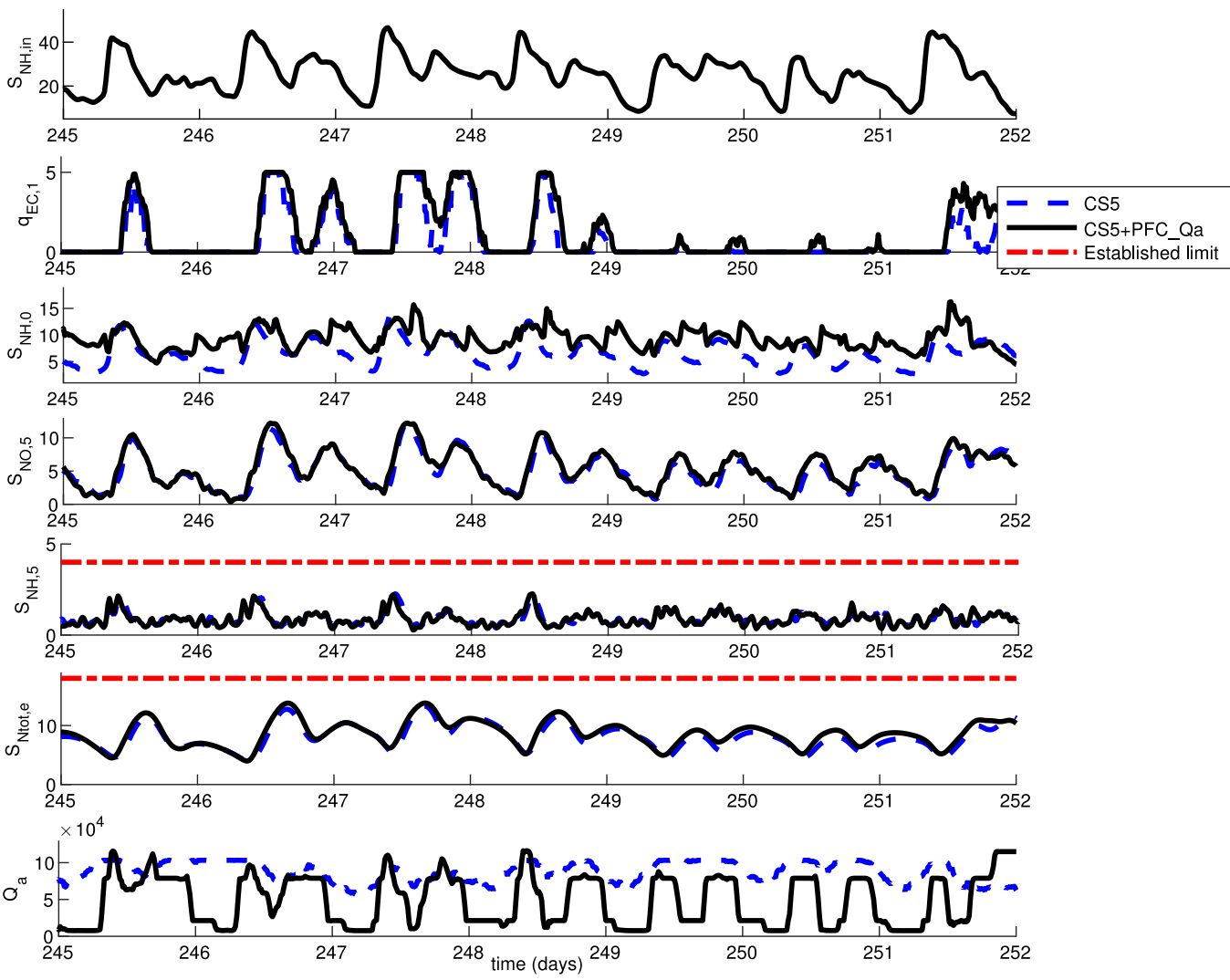

Fig. 20. Time evolution of $S_{\mathrm{NH}, \text { in }}, q_{\mathrm{EC}, 1}, S_{\mathrm{NH}, 0}, S_{\mathrm{NO}, 5}, S_{\mathrm{NH}, 5}, S_{\mathrm{Ntot}, \mathrm{e}}$ and $Q_{\mathrm{a}}$ during one week in summer for CS5 and CS5+PFC_ $Q_{\mathrm{a}}$. 

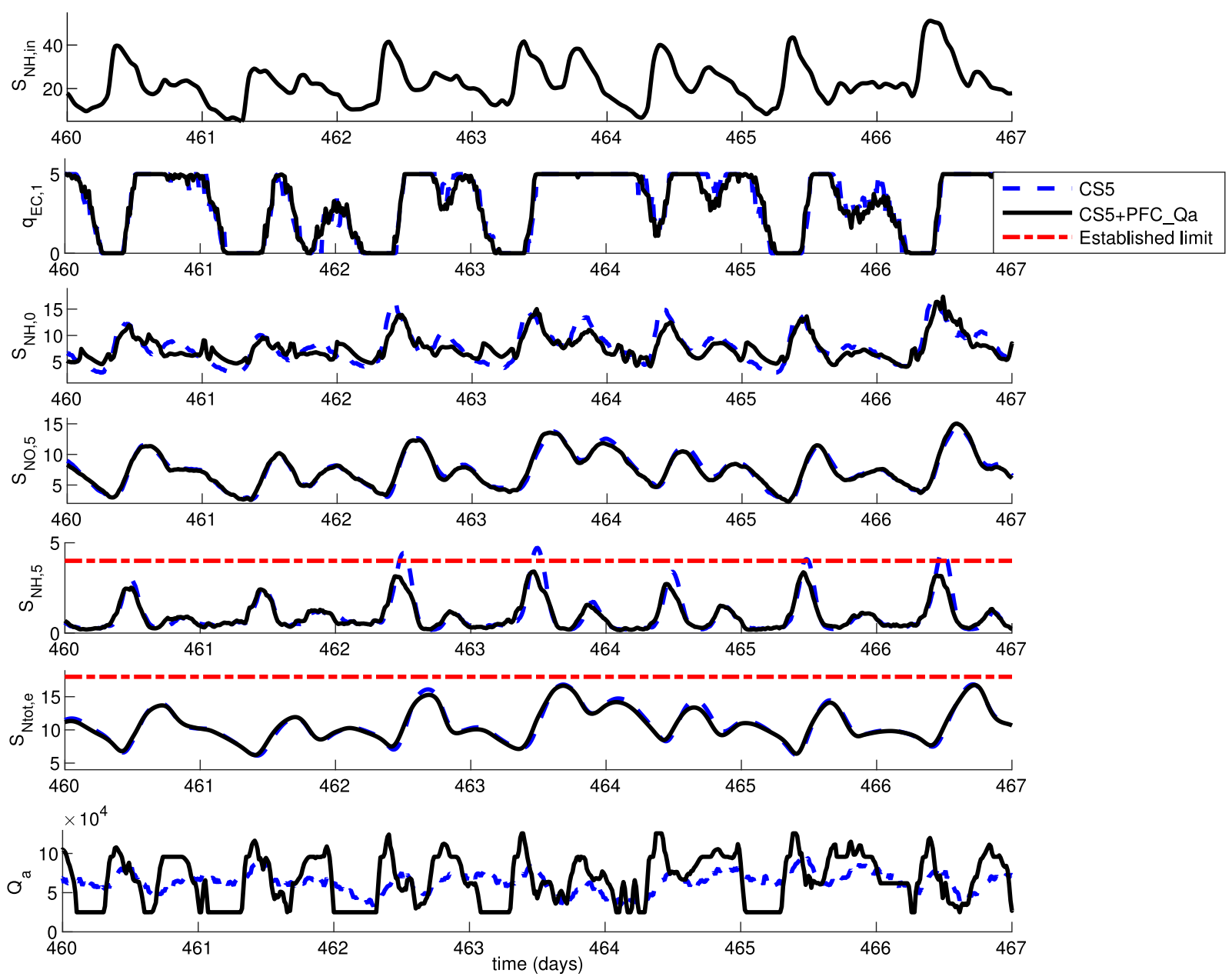

Fig. 21. Time evolution of $S_{\mathrm{NH}, \text { in }}, q_{\mathrm{EC}, 1}, S_{\mathrm{NH}, 0}, S_{\mathrm{NO}, 5}, S_{\mathrm{NH}, 5}, S_{\mathrm{Ntot}, \mathrm{e}}$ and $Q_{\mathrm{a}}$ during one week in winter for CS5 and CS5+PFC_ $Q_{\mathrm{a}}$.

with fixed $S_{0}$ set-point, the fuzzy controller design should be modified by adding $S_{0}$ as input.

$Q_{\mathrm{a}}$ values depend on $T_{\mathrm{as}}$, with both dry and rainy weather, because $T_{\text {as }}$ affects the denitrification and nitrification processes. When $T_{\text {as }}$ decreases, these processes worsen, as shown in the equations of observed conversion rates ((2), (1), (5), (6), (4) and (7)). Therefore, $Q_{a}$ is higher when $T_{\mathrm{as}}$ is lower with the aim of diluting more $S_{\mathrm{NH}, 0}$ (Fig. $3 \mathrm{~b}$ ).

$Q_{a}$ reduction is always limited by $S_{\mathrm{NH}, 0}$. When it is not necessary to dilute $S_{\mathrm{NH}}, Q_{\mathrm{a}}$ is reduced to reduce pumping costs and to improve nitrification and denitrification processes. The $S_{\mathrm{NH}, 0}$ value limits the $Q_{a}$ reduction, since there must be a minimum $S_{\mathrm{NH}}$ dilution in order to make possible to reduce it in the nitrification process to levels below the established limits (Fig. 3c).

Finally, $S_{\mathrm{NH}, 5}$ always plays a priority role in the rules, so that the $Q_{\mathrm{a}}$ increase is always limited by it, because when $S_{\mathrm{NH}, 5}$ is high, $Q_{\mathrm{a}}$ is low or very low (depending on $T_{\mathrm{as}}$ ) (Fig. $3 \mathrm{~d}$ ).

In the case of taking into account all the inputs in all the rules with the condition "is", the minimum number of rules should be 486 to always assign a value to the output. However, due to the priorities of different situations, there are rules where the value given to the output does not depend on some inputs and there are cases where the input condition is "is not" instead of "is". This fact results in a reduction of the computational complexity, and a value is always assigned to the output with only 30 rules. The cases where some inputs are not included in the rules or the condition "is not" is established are explained below:
- $S_{\mathrm{NH}, 5}$ input:

- During dry weather, when $S_{\mathrm{NH}, \text { in }}$ "is Low", while $S_{\mathrm{NH}, 0}$ "is not High" $Q_{a}$ is defined as "Low" or "Very Low" (depending on $\mathrm{T}_{\mathrm{as}}$ ) and the $S_{\mathrm{NH}, 5}$ input is not taken into account because it is restrictive when its value is "High" and when that happens $Q_{a}$ must be "Low" or "Very Low" (depending also on $\mathrm{T}_{\mathrm{as}}$ ).

- When $Q_{\mathrm{a}}$ is assigned a higher value than "Low" or "Very Low", it is restricted to $\mathrm{S}_{\mathrm{NH}, 5}$ "is not High".

- $S_{\mathrm{NO}, 5}$ input:

- For the same reason as $S_{\mathrm{NH}, 5}$ input explained above, during dry weather, when $S_{\mathrm{NH} \text {,in }}$ "is Low", while $S_{\mathrm{NH}, 0}$ "is not High", the $S_{\mathrm{NO}, 5}$ input is not taken into account.

- The $S_{\mathrm{NO}, 5}$ input is neither taken into account when $S_{\mathrm{NH}, 5}$ "is High", and then $Q_{\mathrm{a}}$ is "Low" or "Very Low".

- When $S_{\mathrm{NH}, 0}$ "is not Low", $Q_{\mathrm{a}}$ is increased with the restriction of $S_{\mathrm{NO}, 5}$ "is not High".

- $S_{\mathrm{NH}, 0}$ input:

- $S_{\mathrm{NH}, 0}$ restriction is given when it increases to dilute $S_{\mathrm{NH}}$. Then, if $Q_{a}$ is increased due to a rain event, $S_{\mathrm{NH}, 0}$ does not need to be taken into account.

- $S_{\mathrm{NH}, 5}$ and $S_{\mathrm{NO}, 5}$ are more restrictive than $S_{\mathrm{NH}, 0}$. Therefore, if some of them are "High", $Q_{a}$ is decreased without taken into account $S_{\mathrm{NH}, 0}$ 
- During dry weather, when $\mathrm{T}_{\mathrm{as}}$ is "High" and the situation is not of $S_{\mathrm{NH}, 5}$ or $S_{\mathrm{NO}, 5}$ "High", the decrease of $Q_{a}$ is restricted to the condition of $S_{\mathrm{NH}, 0}$ "is not High".

- $S_{\mathrm{NH}, 0}$ input:

- When $Q_{\mathrm{a}}$ is increased due to a $S_{\mathrm{NH}, 0}$ increase, $S_{\mathrm{NH}, \text { in }}$ input is not taken into account

- When $Q_{\mathrm{a}}$ is decreased because $S_{\mathrm{NH}, 5}$ "is High", $S_{\mathrm{NH}, \text { in }}$ input is neither taken into account

- $Q_{\text {in }}$ input:

- If $S_{\mathrm{NH}, \text { in }}$ "is Low" and $S_{\mathrm{NO}, 5}$ "is High" $Q_{\mathrm{a}}$ is "Low" or "Very Low" (depending on $\mathrm{T}_{\mathrm{as}}$ ) without taking into account $Q_{\text {in }}$ input

- When $Q_{a}$ is decreased because $S_{\mathrm{NH}, 5}$ "is High", $Q_{\text {in }}$ input is neither taken into account

- When $S_{\mathrm{NH} \text {,in }}$ is "Medium" or High", $Q_{\mathrm{a}}$ value depends on $S_{\mathrm{NO}, 5}$ and $\mathrm{T}_{\mathrm{as}}$ inputs and restricted to $Q_{\text {in }}$ "is not High".

- $T_{\mathrm{as}}$ input:

- If a rain event is considered, the $Q_{a}$ value does not depend on $T_{\mathrm{as}}$ input

Regarding the computational complexity of PFC_ $Q_{a}$, the parameters that most influence it are shown in Table A.9. As specified in Kim et al. [33], the number of rules greatly affect the number of operations. Therefore, the reduction of the number of rules to 30 instead of 486 has an important effect on reducing the number of operations and consequently on computational complexity.

The rest of the parameters in Table A.9 do not excessively affect the fuzzy controller complexity and not cause any computation time problem, which is confirmed by the simulation times, shown in Section 4.

\subsection{Operation strategies where PFC_ $Q_{a}$ is tested}

PFC_ $Q_{a}$ is applied in five already tested and published operation strategies. The objective is to modify only the $Q_{a}$ manipulation and compare the results obtained in different scenarios, as shown in Fig. 4. The operation strategies with which the fuzzy controller has been tested are detailed below.

As mentioned in Section 1, Serralta et al. [6], Sadeghassadi et al. [18], Qiao et al. [17], Caraman et al. [20], Revollar et al. [16] and Zhang et al. [19] also apply operation strategies that regulate $Q_{a}$, among other control approaches. However, none of them use BSM2 as working scenario and the controllers should be adapted to the new plant's requirements. In addition, although Serralta et al. [6], Sadeghassadi et al. [18], Qiao et al. [17] and Caraman et al. [20] apply different control techniques, the control approach to manipulate $Q_{\mathrm{a}}$ is the $S_{\mathrm{NO}}$ control, which is already applied in three operation strategies where PFC_ $Q_{\mathrm{a}}$ is tested. In Revollar et al. [16] and Zhang et al. [19], MPC is applied to regulate both $K_{\mathrm{L}} a$ and $Q_{\mathrm{a}}$, and it is not possible to replace only the $Q_{\mathrm{a}}$ manipulation without modifying the controller.

\subsubsection{Default control strategy with $S_{0}$ hierarchical control (CS1)}

The original BSM2 definition (Jeppsson et al. [28]) proposes a Default Control Strategy (DCS). The closed-loop control configuration consists of a Proportional-Integral (PI) controller that controls the $S_{0}$ in the fourth reactor $\left(S_{0,4}\right)$ at a set-point of $2 \mathrm{mg} / \mathrm{l}$ by $K_{\mathrm{L}} a$ in the third tank $\left(K_{\mathrm{L}} a_{3}\right), K_{\mathrm{L}} a$ in the fourth tank $\left(K_{\mathrm{L}} a_{4}\right)$ and $K_{\mathrm{L}} a$ in the fifth tank $\left(K_{\mathrm{L}} a_{5}\right)$ with $K_{\mathrm{L}} a_{5}$ set to the half value of $K_{\mathrm{L}} a_{3}$ and $K_{\mathrm{L}} a_{4} . q_{\mathrm{EC}}$ in the first reactor $\left(q_{\mathrm{EC}, 1}\right)$ is added at a constant flow rate of $2 \mathrm{~m}^{3} / \mathrm{d}$. Two different $Q_{w}$ values are imposed depending on time of the year: from 0 to 180 days and from 364 to 454 days $Q_{w}$ is set to $300 \mathrm{~m}^{3} / \mathrm{d}$; and for the remaining time periods $Q_{w}$ is set to $450 \mathrm{~m}^{3} / \mathrm{d}$. Finally, $Q_{\mathrm{a}}$ is kept fixed to $61,944 \mathrm{~m}^{3} / \mathrm{d}$.

As explained in Section 3.2, PFC_ $Q_{a}$ is designed for control strategies with regulated $S_{0}$ set-point. For this reason, a hierarchical control for $S_{0,4}$ set-point manipulation is added to DCS by means of a fuzzy controller designed and tested in Santín et al. [14] for BSM1 and adapted to the BSM2 characteristics in Santín et al. [22]. This referred paper shows that better results are obtained by regulating $S_{\mathrm{O}}$ based on $S_{\mathrm{NH}}$, instead of trying to keep $S_{O}$ at a fixed set-point.

For testing PFC_ $Q_{a}$, it has been added to this control strategy, to regulate $Q_{a}$, instead of keeping it fixed (CS1+PFC_ $Q_{a}$ ) (Fig. 4a).

\subsubsection{Higher level fuzzy controller in Santín et al. [14] (CS2)}

The control strategy applied in Santín et al. [14] is based on three Model Predictive Control (MPC) controllers and a fuzzy controller. A MPC controls the $S_{0,5}$ set-point by manipulating KLa5 and another MPC controls the $S_{0,4}$ set-point by manipulating $K_{\mathrm{L}} a_{3}$, and $K_{\mathrm{L}} a_{4}$. The fuzzy controller is the same as the one applied in CS1, which manipulates the $S_{0,4}$ and $S_{0,5}$ set-points (with $S_{0,5}$ set-point set to half of the value of $S_{0,4}$ set-point). As in CS1, the hierarchical fuzzy controller configuration is the one established in Santín et al. [22] because it is adapted to BSM2. The third MPC introduces the greatest difference with respect to CS1, controlling $S_{\mathrm{NO}}$ at the output of the second reactor $\left(S_{\mathrm{NO}, 2}\right)$ at a set-point of $2 \mathrm{mg} / \mathrm{l}$ by manipulating $Q_{a}$, instead of keeping $Q_{a}$ constant. The values of $Q_{w}$ and $q_{\mathrm{EC}, 1}$ are the same as in DCS.

PFC $Q_{a}$ is applied replacing the MPC that controls $S_{\mathrm{NO}, 2}$ $\left(\mathrm{CS} 2+P F C_{-} Q_{a}\right)$ (Fig. 4b).

\subsubsection{Control strategy WL-S2 in Barbu et al. [4] (CS3)}

The WL-S2 control strategy of Barbu et al. [4] applies three PI controllers. As in DCS, one PI controller controls SO,4 by manipulating $K_{\mathrm{L}} a_{3}, K_{\mathrm{L}} a_{4}$ and $K_{\mathrm{L}} a_{5}$, with $K_{\mathrm{L}} a_{5}$ set to half of the value of $K_{\mathrm{L}} a_{3}$ and $K_{\mathrm{L}} a_{4}$. Another PI controller tries to keep $S_{\mathrm{NH}, 5}$ at a setpoint of $1 \mathrm{mg} / \mathrm{l}$ by manipulating the $S_{0,4}$ set-point and the last PI controller controls $S_{\mathrm{NO}, 2}$ at a set-point of $1 \mathrm{mg} / \mathrm{l}$ by manipulating $Q_{\mathrm{a}}$. The $Q_{\mathrm{w}}$ and $q_{\mathrm{EC}, 1}$ values are the same as in DCS.

PFC $Q_{a}$ is tested by replacing the $S_{N O, 2}$ control $\left(C S 3+P F C \_Q_{a}\right)$ (Fig. 4c).

\subsubsection{Control strategy WL-S3 in Barbu et al. [4] (CS4)}

The WL-S3 control strategy of Barbu et al. [4] consists of three PI controllers. One PI controller controls $S_{0,4}$ at a fixed set-point of $2 \mathrm{mg} / \mathrm{l}$ by manipulating $K_{\mathrm{L}} a_{3}$ and $K_{\mathrm{L}} a_{4}$. Another PI controller controls $S_{\mathrm{NH}, 5}$ at a fixed set-point of $1 \mathrm{mg} / \mathrm{l}$ by manipulating $K_{\mathrm{L}} a_{5}$. Finally, the third PI controller aims to maintain $S_{\mathrm{NO}, 2}$ at a fixed set-point of $1 \mathrm{mg} / \mathrm{l}$ by manipulating $q_{\mathrm{EC}, 1} \cdot Q_{\mathrm{a}}$ and $Q_{\mathrm{w}}$ maintain the same values as in DCS.

PFC_ $Q_{a}$ is tested with this control strategy by replacing the $Q_{a}$ fixed value of $61944 \mathrm{~m}^{3} / \mathrm{d}\left(\right.$ CS4+PFC_Q $\left.Q_{\mathrm{a}}\right)$ (Fig. 4d).

\subsubsection{Control strategy WL-S4 in Barbu et al. [4] (CS5)}

The WL-S4 control strategy of Barbu et al. [4] applies the same control loops as in CS3 and adds another PI controller to control $S_{\mathrm{NO}, 5}$ at a set-point of $7 \mathrm{mg} / \mathrm{l}$ by manipulating $q_{\mathrm{EC}, 1}$. $Q_{\mathrm{W}}$ maintains the same values as in DCS.

As in CS3, PFC_ $Q_{a}$ is tested by replacing the $S_{\mathrm{NO}, 2}$ control (CS5+PFC_Q $Q_{a}$ ) (Fig. 4e).

\section{Simulation results and discussion}

Table A.11 shows the results obtained with the original operation strategies explained in Section 3.3 and also those obtained by 


\section{Table A.11}

Simulation results of CS1, CS2, CS3, CS4 and CS5 and the same operation strategies adding PFC_Q

\begin{tabular}{|c|c|c|c|c|c|c|c|c|c|c|c|c|c|c|c|c|}
\hline $\begin{array}{l}\text { Evaluation } \\
\text { Criteria }\end{array}$ & & CS1 & CS1+PFC_Qa & $\begin{array}{l}\text { \% of im- } \\
\text { provement }\end{array}$ & CS2 & CS2+PFC_Qa & $\begin{array}{l}\text { \% of im- } \\
\text { provement }\end{array}$ & CS3 & CS3+PFC_Qa & $\begin{array}{l}\text { \% of im- } \\
\text { provement }\end{array}$ & CS4 & CS4+PFC_Qa & $\begin{array}{l}\text { \% of im- } \\
\text { provement }\end{array}$ & CS5 & CS5+PFC_Qa & $\begin{array}{l}\text { \% of im- } \\
\text { provement }\end{array}$ \\
\hline \multirow[t]{5}{*}{ Effluent Quality } & $\begin{array}{l}S_{\text {Ntot,e e limits }} \\
\text { violations (\% of } \\
\text { time) }\end{array}$ & 0.309 & 0.237 & 23.301 & 0.255 & 0.246 & 3.529 & 0.607 & 0.529 & 12.850 & 0.252 & 0.106 & 57.937 & 0.149 & 0.114 & 23.392 \\
\hline & $\begin{array}{l}S_{\mathrm{NH}, \mathrm{e}} \text { limits } \\
\text { violations (\% of } \\
\text { time) }\end{array}$ & 0.229 & 0.0715 & 68.777 & 0.134 & 0.06 & 55.224 & 0.335 & 0.069 & 79.493 & 0.338 & 0.080 & 76.293 & 0.352 & 0.071 & 79.687 \\
\hline & $\begin{array}{l}\text { CD limits } \\
\text { violations (\% of } \\
\text { time) }\end{array}$ & 0.057 & 0.057 & 0 & 0.057 & 0.057 & 0 & 0.057 & 0.057 & 0 & 0.057 & 0.054 & 4.999 & 0.054 & 0.054 & 0 \\
\hline & $\begin{array}{l}\text { TSS limits } \\
\text { violations (\% of } \\
\text { time) }\end{array}$ & 0.343 & 0.343 & 0 & 0.349 & 0.343 & 1.638 & 0.352 & 0.343 & 2.438 & 0.386 & 0.381 & 1.380 & 0.352 & 0.349 & 0.849 \\
\hline & $\begin{array}{l}\text { BOD } 5 \text { limits } \\
\text { violations (\% of } \\
\text { time) }\end{array}$ & 0.226 & 0.226 & 0 & 0.226 & 0.226 & 0 & 0.226 & 0.226 & 0 & 0.226 & 0.226 & 0 & 0.226 & 0.226 & 0 \\
\hline \multirow[t]{5}{*}{ Operational Costs } & $P E($ (kWh/day) & 445.454 & $\begin{array}{l}427.899 \\
\end{array}$ & 3.941 & 692.241 & 428.803 & 38.056 & $\begin{array}{l}492.031 \\
2707572\end{array}$ & $\begin{array}{l}439.319 \\
\end{array}$ & 10.713 & $\begin{array}{l}445.457 \\
0\end{array}$ & 441.700 & 0.843 & 491.873 & 445.064 & 9.516 \\
\hline & $A E$ (kWh/day) & 3601.86 & 3593.732 & 0.226 & 3596.126 & 3591.647 & 0.125 & 3672.740 & 3663.750 & 0.245 & $\begin{array}{l}2088.459 \\
3989.417\end{array}$ & $\begin{array}{l}2701.251 \\
3961.181\end{array}$ & $\begin{array}{l}0.708 \\
0.727\end{array}$ & $\begin{array}{l}26877.241 \\
3818.602\end{array}$ & $\begin{array}{l}26988.786 \\
3632.194\end{array}$ & $\begin{array}{l}-0.430 \\
-0.376\end{array}$ \\
\hline & $\begin{array}{l}E C \text { (kg CoD/day) } \\
M E(\mathrm{kWh} / \text { day })\end{array}$ & $\begin{array}{l}2400 \\
769.113\end{array}$ & $\begin{array}{l}2400 \\
768.464\end{array}$ & $\begin{array}{l}0 \\
0.084\end{array}$ & $\begin{array}{l}2400 \\
770.524\end{array}$ & $\begin{array}{l}2400 \\
768.773\end{array}$ & $\begin{array}{l}0 \\
0.227\end{array}$ & $\begin{array}{l}2400 \\
776.798\end{array}$ & $\begin{array}{l}2400 \\
778.426\end{array}$ & ${ }_{-0.210}^{0}$ & $\begin{array}{l}2441.519 \\
970.036\end{array}$ & $\begin{array}{l}2293.758 \\
967.605\end{array}$ & $\begin{array}{l}6.052 \\
0.251\end{array}$ & $\begin{array}{l}1989.907 \\
778.694\end{array}$ & $\begin{array}{l}2219.619 \\
779.425\end{array}$ & $\begin{array}{l}-11.544 \\
-0.094\end{array}$ \\
\hline & $\begin{array}{l}H^{H} \text { net } \\
M E T_{\text {prod }}(\mathrm{kg} / \mathrm{kg} / \mathrm{day})\end{array}$ & $\begin{array}{l}0 \\
1086.239\end{array}$ & $\begin{array}{l}0 \\
1085.851\end{array}$ & $\begin{array}{l}0 \\
0.036\end{array}$ & $\begin{array}{l}0 \\
1085.659\end{array}$ & $\begin{array}{l}0 \\
1085.849\end{array}$ & $\begin{array}{l}0 \\
-0.018\end{array}$ & $\begin{array}{l}0 \\
1086.164\end{array}$ & $\begin{array}{l}0 \\
1085.963\end{array}$ & $\begin{array}{l}0 \\
0.019\end{array}$ & $\begin{array}{l}0 \\
1086.911\end{array}$ & $\begin{array}{l}0 \\
1085.214\end{array}$ & $\begin{array}{l}0 \\
0.156\end{array}$ & $\begin{array}{l}0 \\
1082.418\end{array}$ & $\begin{array}{l}0 \\
1084.545\end{array}$ & $\begin{array}{l}0 \\
-0.197\end{array}$ \\
\hline & $\begin{array}{l}\text { OCl } \\
\text { oClaay) }\end{array}$ & 8821.425 & 8.801 .551 & 0.225 & 9072.589 & 8800.853 & 2.995 & 8947.298 & 8892.318 & 0.614 & 9451.240 & 9256.709 & 2.058 & 8446.291 & 8665.387 & -2.594 \\
\hline
\end{tabular}


replacing in these strategies the $Q_{a}$ manipulation by PFC_ $Q_{a}$. Thus, the improvements provided by this controller can be observed.

Regarding the effluent quality, improvements in the reduction of $S_{\mathrm{NH}, \mathrm{e}}$ and $S_{\mathrm{Ntot}, \mathrm{e}}$ limit violations are achieved in all the operation strategies explained in Section 3.3 (from CS1 to CS5) using PFC_ $Q_{a}$. The greatest improvements are obtained in the reduction of $S_{\mathrm{NH}, \mathrm{e}}$ limit violations, from $55.22 \%$ in CS2+ PFC_Q $Q_{a}$ to $79.69 \%$ in CS5+PFC_ $Q_{a}$. The reductions in $S_{\text {Ntot,e }}$ limit violations are lower than in $S_{\mathrm{NH}, \mathrm{e}}$ with a maximum reduction of $57.94 \%$ in CS4+PFC_ $Q_{a}$ and a minimum reduction of $3.53 \%$ in CS2+PFC_ $Q_{a}$. The percentages of time of limit violations of $C O D$, TSS and $B O D_{5}$ remain the same, except minor variations in TSS limit violations in CS2, CS3 and CS4. The number of times of limit violations are also the same. This is understable given that the proposed fuzzy controller is designed only to reduce limit violations of $S_{\mathrm{NH}, \mathrm{e}}$ and $S_{\text {Ntot,e. }}$ It should be mentioned that COD, TSS and $B O D_{5}$ limit violations happen when the bypass is active, due to a significant $Q_{\text {in }}$ increase that the plant cannot assume.

By manipulating $Q_{a}$ with $P F C \_Q_{a}$, costs due to $P E$ are reduced in all the operation strategies described in 3.3. The maximum reduction of $P E$ is $38.06 \%$ in $C S 2+P F C \_Q_{a}$, which results in a reduction of $96,154 \mathrm{kWh}$ in one year. The smallest reduction of $P E$ is obtained in CS4+PFC_ $Q_{a}$, but in this case there is also a reduction of $E C$ of $6.05 \%$. In CS5+PFC_ $Q_{a}$, the $P E$ decrease is $9.52 \%$, at the expense of an $\mathrm{OCI}$ increase of $2.59 \%$, mainly due to an $E C$ increase of $11.54 \%$.

The reasons for the results obtained are discussed below. Specific days have been chosen for the analysis of the PFC_ $Q_{a}$ effects in each one of the operation strategies, where there are $S_{\mathrm{NH}, \mathrm{e}}$ limit violations (Figs. 6, 10 and 13), $S_{\text {Ntot,e }}$ limit violations (Figs. 5, 9, 16 and 19), one summer week (Figs. 7, 11, 14 and 17) and one week in winter (Figs. 8, 12, 15, 18 and 21). The comparison between summer and winter weeks allows to observe the $T_{\text {as }}$ effects on the nitrification and denitrification processes and analyze how the $Q_{\mathrm{a}}$ manipulation can affect the pumping energy costs. In some operation strategies, the winter week has also been used for the analysis of $S_{\mathrm{NH}, \mathrm{e}}$ or $S_{\mathrm{Ntot}, \mathrm{e}}$ limit violations.

To illustrate the computational burden of the PFC_ $Q_{a}$ application, Table A.10 shows the CPU time used and the elapsed time in the 609-day simulation of all the operation strategies with and without PFC_ $Q_{a}$, using a $7 \mathrm{GHz}$. Intel Core i7 processor. These values have been obtained with "cputime" and "tic/toc" functions of Matlab, respectively. As can be seen, in the operation strategies in which PFC_ $Q_{a}$ replaces a fixed Qa value (CS1 and CS4), PFC_ $Q_{a}$ results in an increase in elapsed time and CPU time of between approximately 15 and $19 \mathrm{~min}$ in the 609-day simulation. In the case of CS1, as it is a less complex operation strategy, the simulation time is lower, and the PFC_ $Q_{a}$ application results in a higher percentage of increase, of $24.547 \%$ in elapsed time and $25.534 \%$ in CPU time. In CS4, the percentages of increase are smaller, $3.258 \%$ and $3.291 \%$ in elapsed time and CPU time, respectively. However, in the operation strategies where $P F C Q_{a}$ replaces the nitrate control (CS2, CS3 and CS5), the application of PFC_ $Q_{a}$ results in a decrease in elapsed time and CPU time. In CS2, the percentage of decrease is higher $(9.117 \%$ and $8.437 \%$ in elapsed time and CPU time, respectively) because PFC_ $Q_{a}$ replaces an MPC. In CS3 and CS5, PFC_ $Q_{a}$ replaces PI controllers, but even so, elapsed time and $\mathrm{CPU}$ time are reduced, showing that the proposed method does not result in a large computational burden.

\section{1. $S_{\mathrm{NH}, \mathrm{e}}$ limit violations}

With PFC_ $Q_{a}$, when there is a $S_{\mathrm{NH}}$, in increase, $Q_{\mathrm{a}}$ is increased to dilute $S_{\mathrm{NH}, 0}$. On the other hand, $Q_{\mathrm{a}}$ is reduced when $S_{\mathrm{NH}, 5}$ is high, to improve the nitrification process.
The greatest difference between applying PFC_ $Q_{a}$ and keeping $Q_{a}$ fixed (CS1 and CS4) is the $S_{\mathrm{NH}, 0}$ dilution when $S_{\mathrm{NH}, \text { in }}$ increases. Fig. 6 corresponds to day 368 with CS1 and with CS1+ PFC_ $Q_{a}$. In this day, there is an increase in the $Q_{\text {in }}$ to $S_{\mathrm{NH} \text {,in }}$ ratio that is representative of a rain event and causes a $Q_{a}$ increase, which takes place earlier in CS1+PFC_ $Q_{\mathrm{a}}$. The subsequent $S_{\mathrm{NH}, 5}$ increase results in a $Q_{a}$ decrease in CS1+PFC_ $Q_{a}$, which is reduced to even lower levels than in CS1.

Fig. 18 shows a winter week with CS4 and with CS4+PFC_ $Q_{a}$. The worsening of the nitrification process with low $T_{\text {as }}$ results in $S_{\mathrm{NH}, \mathrm{e}}$ limit violations on days 462,463 and 466 with CS4. There is no rain event during this period, but the $S_{\mathrm{NH} \text {, in }}$ increase produces a $Q_{a}$ increase in CS4+PFC_ $Q_{a}$, which is higher due to low $T_{\text {as }}$. As happens in 6 by CS1+PFC_ $Q_{a}$, the previous $S_{\mathrm{NH}, 0}$ dilution and the subsequent $Q_{a}$ reduction when $S_{\mathrm{NH}, 5}$ increases allows to avoid $S_{\mathrm{NH}, \mathrm{e}}$ limit violations by CS4+PFC_Q $\mathrm{Q}$.

In the case of the operation strategies that manipulate $Q_{a}$ to control $S_{\mathrm{NO}, 2}$ at the set-point of $1 \mathrm{mg} / \mathrm{l}$ (CS2, CS3 and CS5), when $S_{\mathrm{NO}, 5}$ is lower $Q_{\mathrm{a}}$ is increased to increase $S_{\mathrm{NO}}$ in the anoxic zone, and, inversely, when $S_{\mathrm{NO}, 5}$ is greater $Q_{\mathrm{a}}$ is decreased. CS2 applies an MPC with a higher gain than the PI controllers of CS3 and CS5, and the $Q_{a}$ variations are greater. Furthermore, in CS3 and CS5 the value of $Q_{a}$ is limited to $103,240 \mathrm{~m}^{3} / \mathrm{d}$, which is less than the maximum allowed $\left(309,720 \mathrm{~m}^{3} / \mathrm{d}\right)$. In Fig. 10 , it is observed that in CS2, in the period of time when $S_{\mathrm{NO}, 5}$ is reduced and $Q_{a}$ is increased, there is a coincidental increase of $S_{\mathrm{NH}, \text { in }}$, which is diluted thanks to the $Q_{\mathrm{a}}$ increase. Although on this day $Q_{a}$ is increased a little more in CS2+PFC_ $Q_{a}$ than in CS2, this is not the main difference between both, but the subsequent $Q_{a}$ decrease when $S_{N H, 5}$ increases. In CS2 $Q_{a}$ is decreased later than in CS2+PFC_ $Q_{\mathrm{a}}$ because it is caused by the $S_{\mathrm{NO}, 5}$ increase and not by the $S_{\mathrm{NH}, 5}$ increase. Finally, the $S_{\mathrm{NH}, \mathrm{e}}$ limit violation that take place on day 435 with CS2 are avoided with CS2+PFC_ $Q_{a}$. It should be noted that CS2 does not aim to dilute $S_{\mathrm{NH}, 0}$ when $S_{\mathrm{NH}, \text { in }}$ increases, but the $Q_{a}$ increase, due to a $S_{\mathrm{NO}, 5}$ decrease, coincides with the $S_{\mathrm{NH} \text {,in }}$ increase due to the influent dynamics. In Fig. 13 and Fig. 19, it is observed how violations in the $S_{\mathrm{NH}, \mathrm{e}}$ limits on day 368 with CS3 and on days 559 and 560 with CS5 are avoided with $C S 3+P F C_{-} Q_{a}$ and $C S 5+P F C_{-} Q_{a}$, respectively. In CS3 and CS5 the $Q_{a}$ variations are lower than in CS2. In these operation strategies, there is an important difference in the $S_{\mathrm{NH}, 0}$ dilution with the $Q_{a}$ increase with respect to CS3+PFC_ $Q_{a}$ and CS5+PFC_ $Q_{a}$, respectively. As in CS2, $Q_{a}$ is reduced later in CS3 and CS5 than in CS3+PFC_ $Q_{a}$ and CS5+PFC_ $Q_{a}$, respectively, and is caused by the increase of $S_{\mathrm{NO}, 5}$ instead of by the increase of $S_{\mathrm{NH}, 5}$.

\section{2. $S_{\text {Ntot,e }}$ limit violations}

As explained in the previous section, when there is a $S_{\mathrm{NH} \text {, in }}$ increase, $Q_{\mathrm{a}}$ is increased to dilute $S_{\mathrm{NH}, 0}$. When $S_{\mathrm{NO}, 5}$ increases, $Q_{\mathrm{a}}$ is slightly reduced, which improves the denitrification process, which results in a $S_{\mathrm{NO}, 5}$ reduction in a first period of time. However, the reduction of $Q_{a}$ also improves the nitrification process, whose effect in $S_{\mathrm{NO}, 5}$ depends on the $S_{\mathrm{O}}$ required. It should be noted that an excessive $Q_{a}$ reduction would probably cause a subsequent $S_{\mathrm{NO}, 5}$ increase by improving the nitrification process.

As previously discussed, there is a reduction of $S_{\text {Ntot,e }}$ limit violations by applying PFC_ $Q_{a}$ in all tested operation strategies, but these reductions are lower than the reductions of $S_{\mathrm{NH}, \mathrm{e}}$ limit violations. The reason for this is that when there is a simultaneous increase in $S_{\mathrm{Ntot}, \mathrm{e}}$ and $S_{\mathrm{NH}, 5}$, the $S_{\mathrm{NH}, 5}$ increase requires a stronger and earlier $Q_{\mathrm{a}}$ reduction, which is detrimental to reduce the $S_{\mathrm{NO}, 5}$ peak. The best option is to reduce $Q_{a}$ when $S_{\mathrm{NO}, 5}$ is greater, and thus to reduce the $S_{\mathrm{NO}, 5}$ peak to improve the denitrification process without an excessive generation of $S_{\mathrm{NO}, 5}$ by improving nitrification. 
In Fig. 5 and in Fig. 16, the effects on $S_{\text {Ntot, e }}$ by applying PFC_ $Q_{a}$ can be compared to those obtained by keeping $Q_{\mathrm{a}}$ fixed. As with $S_{\mathrm{NH}, \mathrm{e}}$ limit violation reduction, the main difference in this case is the $Q_{\mathrm{a}}$ increase, when $S_{\mathrm{NH}, \text { in }}$ increases, to dilute $S_{\mathrm{NH}, 0}$. Although the $S_{\mathrm{NH}, 5}$ increase results in $Q_{\mathrm{a}}$ decrease before $S_{\mathrm{NO}, 5}$ reaches high values, Fig. 5 and Fig. 16 show how CS1+PFC_ $Q_{a}$ and CS4+PFC_ $Q_{a}$ avoid $S_{\text {Ntot,e }}$ limit violation on day 341 unlike CS1 and CS4.

The $Q_{\mathrm{a}}$ effect in CS3 and in CS5 is similar to that obtained in CS1 and CS4. As explained in Section 4.1, although there is a $Q_{a}$ regulation to control $S_{\mathrm{NO}, 2}$, the low gain of the PI controllers in CS1 and CS4 results in a smaller and slower $Q_{a}$ increase when $S_{\mathrm{NO}, 5}$ decreases, which in turn results in a lower dilution of $S_{\mathrm{NH}, 0}$. This fact is observed in Fig. 15 where $S_{\text {Ntot,e }}$ limit violations with CS3 on days 463,464 and 466 are avoided with CS3+PFC_Q $Q_{a}$.

In addition, controlling $S_{\mathrm{NO}, 2}$ by manipulating $Q_{\mathrm{a}}$ in CS2, CS3, and CS5 has another detrimental effect on $S_{\mathrm{Ntot}, \mathrm{e}}$ limit violations when there is a long rain period. In these cases, the denitrification process worsens due to a HRT decrease, and the $S_{\mathrm{NO}}$ level increases. The $S_{\mathrm{NO}, 2}$ control excessively reduces $Q_{a}$, which improves the denitrification process, but also improves the nitrification process, generating more $S_{\mathrm{NO}}$. $Q_{\mathrm{a}}$ decrease affects especially $S_{\mathrm{NH}, 0}$ that is increased. This effect can be seen, for instance, in Fig. 9 and in Fig. 19.

In the case of CS2+PFC_ $Q_{a}$, the reduction of $S_{\text {Ntot, e }}$ limit violations with respect to CS2 is lower than in the other operation strategies because CS2 dilutes $S_{\mathrm{NH}, 0}$ when there is a $S_{\mathrm{NH} \text {,in }}$ increase. In addition, the subsequent reduction of $Q_{a}$ is performed when $S_{\mathrm{NO}, 5}$ is greater since it does not take into account $S_{\mathrm{NH}, 5}$. However, CS2 has an increase in $S_{\mathrm{Ntot}, \mathrm{e}}$ limit violations mainly due to long rain periods.

\subsection{Operational costs}

The cost reduction is mainly due to the saving in pumping energy thanks to a lower average $Q_{a}$. It occurs mainly in summer when $T_{\text {as }}$ is high, nitrification and denitrification processes improve and therefore the $S_{\mathrm{NH}, 0}$ dilution to keep both $S_{\mathrm{Ntot}, \mathrm{e}}$ and $S_{\mathrm{NH}, \mathrm{e}}$ below the established limits is less necessary. The $Q_{\mathrm{a}}$ regulation has the objective of reducing the $S_{\mathrm{NH}, \mathrm{e}}$ and $S_{\mathrm{Ntot}, \mathrm{e}}$ peaks although the $S_{\mathrm{NH}, 0}$ dilution is lower.

Figs. 7, 11, 14, 17 and 20 show how most of the time $Q_{a}$ is lower with the PFC_ $Q_{a}$ than with the original control strategies. This difference is greater in CS2, CS3 and CS5, which manipulate $Q_{\text {a }}$ to control $S_{\mathrm{NO}, 2}$, coinciding with the PE costs in Table A.11. In these control strategies, $Q_{a}$ is increased more in summer than in winter because $S_{\mathrm{NO}, 5}$ is lower and it is necessary to recirculate more $S_{\mathrm{NO}}$ to maintain $S_{\mathrm{NO}, 2}$ at the set-point of $1 \mathrm{mg} / \mathrm{l}$. However, in summer, this $Q_{\mathrm{a}}$ increase is unnecessary to keep $S_{\mathrm{NH}, \mathrm{e}}$ and $S_{\mathrm{Ntot}, \mathrm{e}}$ below the limits. The $Q_{a}$ increase is greater in CS2 than in CS3 and CS5 because the maximum $Q_{a}$ limit is greater. This fact also results in higher PE costs, shown in Table A.11.

Figs. 8, 12, 15, 18 and 21 show a week in which $T_{\text {as }}$ is low. As in this case the nitrification and denitrification processes worsen, PFC_ $Q_{a}$ increases more $Q_{a}$ in order to dilute $S_{\mathrm{NH}, 0}$ when $S_{\mathrm{NH}, \text { in }}$ increases. The $S_{\mathrm{Ntot}, \mathrm{e}}$ and $S_{\mathrm{NH}, \mathrm{e}}$ peaks are higher than in summer and closer to the established limits. In fact, there are $S_{\mathrm{NH}, \mathrm{e}}$ limit violations in CS1, CS3, CS4 and CS5 and $S_{\text {Ntot,e }}$ limit violations in CS3, while with the application of PFC $Q_{a}$ they are avoided. In all tested operation strategies, except in CS2, the $Q_{a}$ average in winter is higher with the application of PFC_ $Q_{a}$, but the $Q_{a}$ reduction in summer, explained above, results in a reduction of $P E$ annual costs.

In CS5+PFC_ $Q_{a}$ compared to CS5, although there is a decrease of $P E$ costs, there is an $\mathrm{OCI}$ increase mainly due to an increase of EC costs. CS5 manipulates $q_{\mathrm{EC}, 1}$ to control $S_{\mathrm{NO}, 5}$ at a set-point of 7 $\mathrm{mg} / \mathrm{l}$. The application of PFC_ $Q_{a}$ reduces $Q_{a}$ in summer most of the time to reduce $P E$ costs because the values of $S_{\mathrm{Ntot}, \mathrm{e}}$ and $S_{\mathrm{NH}, \mathrm{e}}$ are far from the established limits. However, in CS5+PFC_ $Q_{a}$, when $S_{\mathrm{NO}, 5}$ is greater than $7 \mathrm{mg} / \mathrm{l}$, there is a higher $q_{\mathrm{EC}, 1}$ increase than in CS5, although $S_{\mathrm{NO}, 5}$ values are not considered dangerous for a possible $S_{\text {Ntot,e }}$ limit violation, as can be observed in Fig. 20.

In CS4, there is also a $q_{\mathrm{EC}, 1}$ regulation, but in this case to control $S_{\mathrm{NO}, 2}$, instead of $S_{\mathrm{NO}, 5}$, at the set-point of $1 \mathrm{mg} / \mathrm{l}$. As in CS4+PFC_Q $Q_{a} Q$ a is usually lower than in CS4, $S_{\mathrm{NO}, 2}$ is also lower and therefore less $q_{\mathrm{EC}, 1}$ is added, as can be seen in Fig. 17. Thus, $E C$ costs are reduced by CS4+PFC_ $Q_{a}$ compared to CS4.

It must be taken into account that the PFC_ $Q_{a}$ application requires the addition of sensors for the $S_{\mathrm{NH}, \text { in }}, S_{\mathrm{NH}, 0}, S_{\mathrm{NO}, 5}, T_{\text {as }}$ and $Q_{\text {in }}$ measurements to the operation strategies, in which PFC_ $Q_{a}$ is tested, except in CS5 that already uses a $S_{\mathrm{NO}, 5}$ sensor. These sensors are commonly used in WWTPs and do not require excessive investment. With only the reduction of pumping energy costs, the investment would be recovered in less than a year in CS2 and a little more time in the rest. If the reduction of fines due to the reduction of nutrient limit violations is taken into account, the return on investment would be in a shorter time. In addition, regardless of the PFC_ $Q_{a}$ application, the added sensors provide important information to the plant. Specifically, the $T_{\mathrm{as}}$, $Q_{\text {in }}$ and $S_{\mathrm{NH} \text {,in }}$ measurements are quite essential, and it is very common to have these sensors, even though they are not used in the operation strategy.

\section{Conclusions}

This article has presented a new control strategy to manipulate $Q_{a}$ taking into account its effects in the different areas of the biological treatment of a wastewater treatment plant, by applying a fuzzy controller. PFC_ $Q_{a}$ has been tested in five already tested and published operation strategies, replacing the original $Q_{a}$ manipulation and comparing results. The PFC_ $Q_{a}$ application has reduced $S_{\mathrm{NH}, \mathrm{e}}$ and $S_{\mathrm{Ntot}, \mathrm{e}}$ limit violations and $P E$ costs in all tested operation strategies. The only exception is CS5, where there is an $\mathrm{OCI}$ increase due to a $q_{\mathrm{EC}, 1}$ increase. The graphs of evolution over time of the most important concentrations show the effects of $Q_{a}$, corroborating that the objectives of PFC_ $Q_{a}$ are met . The main conclusions of the PFC_ $Q_{a}$ application and its comparison with the original control strategies are:

- The $Q_{\mathrm{a}}$ increase to dilute $S_{\mathrm{NH}, 0}$, when $S_{\mathrm{NH}, \text { in }}$ or $Q_{\text {in }}$ increases, has an important effect in reducing $S_{\mathrm{Ntot}, \mathrm{e}}$ and $S_{\mathrm{NH}, \mathrm{e}}$ peaks to avoid limit violations

- The $Q_{\mathrm{a}}$ decrease, when it is not necessary to dilute $S_{\mathrm{NH}, 0}$ due to low $S_{\mathrm{NH} \text {, in }}$ levels and especially in summer, reduces $P E$ costs.

- The $Q_{\mathrm{a}}$ reduction, when $S_{\mathrm{NH}, 5}$ increases, generates a HRT increase that improves the nitrification process, reducing the $S_{\mathrm{NH}, \mathrm{e}}$ peaks and avoiding limit violations

- The $Q_{a}$ reduction, when $S_{N O, 5}$ increases, reduces it in a first period of time due to the denitrification process improvement, which allows to avoid $S_{\text {Ntot,e }}$ limit violations. However, a large and/or too premature $Q_{\mathrm{a}}$ reduction can generate a subsequent increase of $S_{\mathrm{NO}, 5}$. A fact that happens in the PFC_ $Q_{a}$ application if there is a large increase in $S_{\mathrm{NH}, 5}$ and $S_{\mathrm{NO}, 5}$ on the same day.

- Keeping $Q_{\mathrm{a}}$ fixed, as in CS1 and CS4, results in a poor $S_{\mathrm{NH}, 0}$ dilution when $S_{\mathrm{NH} \text {,in }}$ or $Q_{\text {in }}$ increase and does not improve the nitrification process when the $S_{\mathrm{NH}, 5}$ increase requires it. The average value of $Q_{a}$ in summer is excessively high, which increases $P E$ costs.

- The $Q_{a}$ manipulation to control $S_{\mathrm{NO}, 2}$, as in CS2, CS3 and CS5, excessively increases $Q_{a}$ in summer, when less dilution is required, to try to keep $S_{\mathrm{NO}, 2}$ at the set-point of $1 \mathrm{mg} / \mathrm{l}$, 
generating an increase of $P E$ costs. When there is an increase in $S_{\mathrm{NH}, 5}, Q_{\mathrm{a}}$ is not reduced, and during long rain periods $Q_{\mathrm{a}}$ is excessively reduced.

- The combination of the PFC_Q $Q_{a}$ application with the $S_{\mathrm{NO}, 5}$ control by manipulating $q_{\mathrm{EC}, 1}$ can generate increases of $E C$ costs in the summer period, especially with low $S_{\mathrm{NO}, 5}$ setpoints.

- The combination of the PFC_ $Q_{a}$ application with the $S_{\mathrm{NO}, 5}$ control by manipulating $q_{\mathrm{EC}, 1}$ can generate increases of $E C$ costs in the summer period, especially with low $S_{\mathrm{NO}, 5}$ setpoints.

It is worth mentioning that $Q_{a}$ has effects on the amount of $S_{0}$ in the aerobic zone and therefore also on nitrous oxide emissions. However, the current PFC_ $Q_{a}$ has not been designed to take into account nitrous oxide emissions or any other greenhouse gas. Updating the approach of the $Q_{a}$ regulation to take this effect into account is planned as future work.

Also, in the case of WWTPs that carry out the biological phosphorus removal, the effects of PFC_ $Q_{a}$ application must be analyzed.

Finally, the present work constitutes the first step of the study to be completed by further tests in pilot or real plants.

\section{Declaration of competing interest}

The authors declare that they have no known competing financial interests or personal relationships that could have appeared to influence the work reported in this paper.

\section{Acknowledgments}

This work was partially supported by the Spanish MICINN program under grants DPI-2016-77271-R and PID2019-105434RBC33.

Marian Barbu acknowledge the support of the project "EXPERT", Contract no. 14PFE/17.10.2018.

\section{Appendix}

\footnotetext{
[System ]

Name $=$ 'PFC_Qa'

Type $=$ ' mamdani'

Version $=2.0$

NumInputs $=6$

NumOutputs $=1$

NumRules $=30$

AndMethod = ' $\min$ '

OrMethod = ' $m a x$ '

ImpMethod = ' $\mathrm{min}$ '

AggMethod = ' $\max$ '

DefuzzMethod = ' centroid '

[ Input 1]

Name $=$ 'SNHin '

Range $=\left[\begin{array}{ll}12 & 40\end{array}\right]$

NumMFs $=3$

MF1= 'Low' : ' zmf' , [ 12 24.2]

MF2= 'Medium': ' trimf ', , [18 25 32]

MF3= 'High ' : ' smf' , [ [ 26 40]

[ Input2 ]

Name $=$ 'SNHO'

Range $=\left[\begin{array}{ll}10 & 21\end{array}\right]$
}

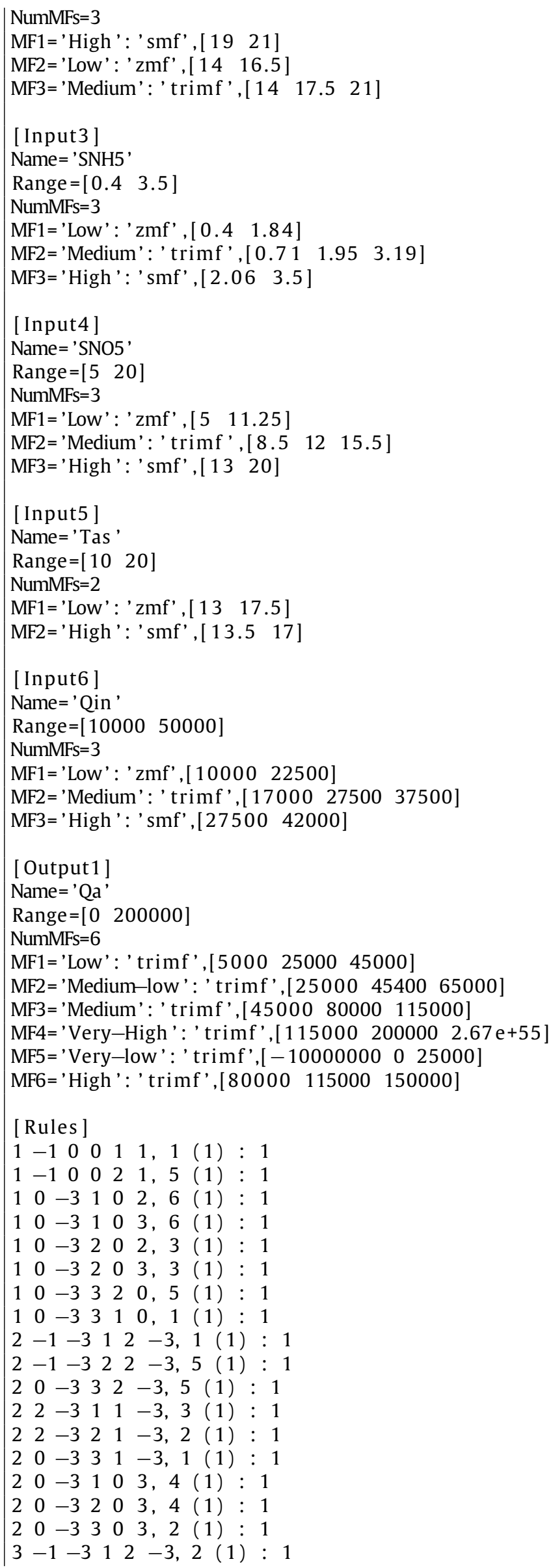




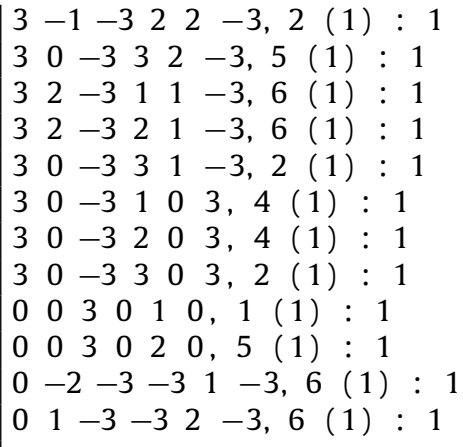

\section{References}

[1] Katebi R, Johnson MA, Wilkie J. Control and instrumentation for wastewater treatment plants. Springer Science \& Business Media; 2012.

[2] Santín I, Pedret C, Vilanova R. Control and decision strategies in wastewater treatment plants for operation improvement, vol. 86. Springer; 2016.

[3] i Arbós RV, López IS, Pedret C. Control and operation of wastewater treatment plants: Challenges and state of the art. Rev Iberoam Autom Inform Ind (RIAI) 2017;14(4):329-45.

[4] Barbu M, Santin I, Vilanova R. Applying control actions for water line and sludge line to increase wastewater treatment plant performance. Ind Eng Chem Res 2018;57(16):5630-8

[5] Santín I, Vilanova R, Barbu M, Pedret C. Manipulating the sludge flow to increase methane production and to reduce effluent pollution in wastewater treatment plants. In: 2016 24th Mediterranean conference on control and automation. IEEE; 2016, p. 877-82.

[6] Serralta J, Ribes J, Seco A, Ferrer J. A supervisory control system for optimising nitrogen removal and aeration energy consumption in wastewater treatment plants. Water Sci Technol 2002;45(4-5):309-16.

[7] Belchior CAC, Araújo RAM, Landeck JAC. Dissolved oxygen control of the activated sludge wastewater treatment process using stable adaptive fuzzy control. Comput Chem Eng 2012;37:152-62.

[8] Yang T, Qiu W, Ma Y, Chadli M, Zhang L. Fuzzy model-based predictive control of dissolved oxygen in activated sludge processes. Neurocomputing 2014;136:88-95.

[9] Harja G, Nascu I, Muresan C, Nascu I. Improvements in dissolved oxygen control of an activated sludge wastewater treatment process. Circuits Systems Signal Process 2016;35(6):2259-81.

[10] Du X, Wang J, Jegatheesan V, Shi G. Dissolved oxygen control in activated sludge process using a neural network-based adaptive pid algorithm. Appl Sci 2018;8(2):261

[11] Santín I, Barbu M, Pedret C, Vilanova R. Dissolved oxygen control in biological wastewater treatments with non-ideal sensors and actuators. Ind Eng Chem Res 2019;58(45):20639-54.

[12] Vega P, Revollar S, Francisco M, Martín J. Integration of set point optimization techniques into nonlinear MPC for improving the operation of WWTPs. Comput Chem Eng 2014;68:78-95.
[13] Błaszkiewicz K, Piotrowski R, Duzinkiewicz K. A model-based improved control of dissolved oxygen concentration in sequencing wastewater batch reactor. Stud Inform Control 2014;23(4):323-32.

[14] Santín I, Pedret C, Vilanova R. Applying variable dissolved oxygen set point in a two level hierarchical control structure to a wastewater treatment process. J Process Control 2015;28:40-55.

[15] Li F, Qiao J, Han H, Yang C. A self-organizing cascade neural network with random weights for nonlinear system modeling. Appl Soft Comput 2016;42:184-93.

[16] Revollar S, Vega P, Vilanova R, Francisco M. Optimal control of wastewater treatment plants using economic-oriented model predictive dynamic strategies. Appl Sci 2017;7(8):813.

[17] Qiao J-F, Hou Y, Zhang L, Han H-G. Adaptive fuzzy neural network control of wastewater treatment process with multiobjective operation. Neurocomputing 2018;275:383-93.

[18] Sadeghassadi M, Macnab CJ, Gopaluni B, Westwick D. Application of neural networks for optimal-setpoint design and MPC control in biological wastewater treatment. Comput Chem Eng 2018;115:150-60.

[19] Zhang A, Yin X, Liu S, Zeng J, Liu J. Distributed economic model predictive control of wastewater treatment plants. Chem Eng Res Des 2019; $141: 144-55$.

[20] Caraman S, Luca L, Vasiliev I, Barbu M. Optimal-setpoint-based control strategy of a wastewater treatment process. Processes 2020;8(10):1203.

[21] Karches T. Effect of internal recirculation on reactor models in wastewater treatment. WIT Trans Ecol Environ 2018;228:145-53.

[22] Santín I, Pedret C, Vilanova R, Meneses M. Advanced decision control system for effluent violations removal in wastewater treatment plants. Control Eng Pract 2016;279:207-19.

[23] Camilleri F, Katebi R. Fuzzy logic control of integrated wastewater systems. IFAC Proc Vol 2005;38(1):161-6.

[24] Boiocchi R, Gernaey KV, Sin G. Understanding $\mathrm{N}_{2} \mathrm{O}$ formation mechanisms through sensitivity analyses using a plant-wide benchmark simulation model. Chem Eng J 2017;317:935-51.

[25] Gernaey K, Jeppsson U, Vanrolleghem P, Copp J. Benchmarking of control strategies for wastewater treatment plants. Scientific and Technical report No. 23, London, UK: IWA Publishing; 2014.

[26] Alex J, Beteau J, Copp J, Hellinga C, Jeppsson U, Marsili-Libelli S, et al. Benchmark for evaluating control strategies in wastewater treatment plants. In: Control conference, 1999 European. IEEE; 1999, p. 3746-51.

[27] Copp JB. The COST simulation benchmark: Description and simulator manual: a product of COST action 624 and COST action 682. EUR-OP; 2002.

[28] Jeppsson U, Pons M-N, Nopens I, Alex J, Copp J, Gernaey K, et al. Benchmark Simulation Model No 2: general protocol and exploratory case studies. Water Sci Technol 2007;56(8):67-78.

[29] Nopens I, Benedetti L, Jeppsson U, Pons M-N, Alex J, Copp JB, et al. Benchmark Simulation Model No 2: finalisation of plant layout and default control strategy. Water Sci Technol 2010;62(9):1967-74.

[30] Henze M, Grady C, Gujer W, Marais G, Matsuo T. Activated sludge model 1. Scientific and Technical report No. 1, London, UK: IAWQ; 1987.

[31] Klir G, Yuan B. Fuzzy sets and fuzzy logic, vol. 4. Prentice Hall New Jersey; 1995.

[32] Mamdani E. Application of fuzzy algorithms for control of simple dynamic plant. Proc Inst Electr Eng 1976;121(12):1585-8.

[33] Kim YH, Ahn SC, Kwon WH. Computational complexity of general fuzzy logic control and its simplification for a loop controller. Fuzzy Sets and Systems 2000;111(2):215-24 